\title{
OPEN A survey of the kinome pharmacopeia reveals multiple scaffolds and targets for the development of novel anthelmintics
}

\author{
Jessica Knox ${ }^{1,2}$, Nicolas Joly ${ }^{3}$, Edmond M. Linossi ${ }^{4}$, José A. Carmona-Negrón ${ }^{4}$, Natalia Jura ${ }^{4,5}$,
} Lionel Pintard ${ }^{3}$, William Zuercher ${ }^{6}$ \& Peter J. Roy ${ }^{1,2,7 凶}$

Over one billion people are currently infected with a parasitic nematode. Symptoms can include anemia, malnutrition, developmental delay, and in severe cases, death. Resistance is emerging to the anthelmintics currently used to treat nematode infection, prompting the need to develop new anthelmintics. Towards this end, we identified a set of kinases that may be targeted in a nematodeselective manner. We first screened 2040 inhibitors of vertebrate kinases for those that impair the model nematode Caenorhabditis elegans. By determining whether the terminal phenotype induced by each kinase inhibitor matched that of the predicted target mutant in C. elegans, we identified 17 druggable nematode kinase targets. Of these, we found that nematode EGFR, MEK1, and PLK1 kinases have diverged from vertebrates within their drug-binding pocket. For each of these targets, we identified small molecule scaffolds that may be further modified to develop nematode-selective inhibitors. Nematode EGFR, MEK1, and PLK1 therefore represent key targets for the development of new anthelmintic medicines.

The burden of parasitic nematodes on humanity is severe. Over one billion people suffer from intestinal nematode infections alone ${ }^{1,2}$. Chronic infection results in a myriad of symptoms including malnutrition, anemia and developmental delay. Left untreated, infections can lead to cognitive impairment that adversely affects education and employment and contributes to a cycle of poverty. Severe infections can lead to death ${ }^{2,3}$. All told, parasitic nematode infections are responsible for an estimated disease burden of over 3.4 million disability-adjusted life years (DALYs), which is equivalent to that of tuberculosis or malaria ${ }^{4}$.

Anthelmintics that are used to treat parasitic nematode infections in humans include albendazole, mebendazole, diethylcarbamazine and ivermectin ${ }^{3}$. Emerging evidence indicates that mass drug administration is driving the evolution of resistance to these anthelmintics within human parasite populations ${ }^{2}$. Parasitic nematodes further impact our wellbeing by parasitizing livestock and crops, leading to billions of dollars in losses annually, increasing food costs and contributing to malnutrition ${ }^{5-8}$. As a result of intensive anthelmintic pressure, nematode parasites of livestock have rapidly developed resistance to every class of anthelmintic applied ${ }^{9-13}$. This reinforces the alarm raised by the emergence of resistance among the nematode parasites of humans. Hence, there is a clear need for the development of new compounds to combat parasitic nematodes of humans, animals, and plants.

Kinases hold potential as targets for the development of novel anthelmintics ${ }^{14-21}$. Kinases are a large family of enzymes that phosphorylate substrates to co-ordinate nearly every signaling pathway in the cell ${ }^{22}$. For example, upon binding of the epidermal growth factor (EGF) ligand, the EGF tyrosine kinase transmembrane receptor

\footnotetext{
${ }^{1}$ Department of Molecular Genetics, University of Toronto, Toronto, ON M5S 1A8, Canada. ${ }^{2}$ The Donnelly Centre for Cellular and Biomolecular Research, University of Toronto, Toronto, ON M5S 3E1, Canada. ${ }^{3}$ Programme Équipe Labellisée Ligue Contre Le Cancer, Institut Jacques Monod, UMR7592, Université de Paris, CNRS, Paris, France. ${ }^{4}$ Cardiovascular Research Institute, University of California San Francisco, San Francisco, CA 94158, USA. ${ }^{5}$ Department of Cellular and Molecular Pharmacology, University of California San Francisco, San Francisco, CA 94158, USA. ${ }^{6}$ School of Pharmacy, UNC Eshelman, University of North Carolina at Chapel Hill, Chapel Hill, NC 27599, USA. ${ }^{7}$ Department of Pharmacology and Toxicology, University of Toronto, Toronto, ON M5S 1A8, Canada. ${ }^{\boxplus}$ email: peter.roy@utoronto.ca
} 
(EGFR) dimerizes and autophosphorylates itself, changing the conformation of its intracellular domain. Adaptor proteins are consequently recruited to initiate a RAS-driven MAP kinase signaling cascade that controls numerous events, including cell survival, proliferation, differentiation, growth, migration, and resistance to apoptosis $^{23,24}$.

Here, we focus on kinases as potential anthelmintic targets for several reasons. First, there is extensive knowledge of kinase structure. The structures of protein kinases are among the most intensely studied of any protein family ${ }^{25}$ (http://www.thesgc.org/science/protein-kinases/); the Protein Data Bank (PDB) contains over 7000 kinase structures with and without inhibitors bound ${ }^{26}$ (http://www.rcsb.org/). The vast number of solved kinase structures is likely due to their key roles in both development and pathogeneses, but also because of the relative ease of kinase purification and in vitro biochemical assays. Given a benchmark of $30 \%$ sequence identity for reliable prediction of a protein structure by homology modeling, models of many nematode kinases can be constructed based on the experimental 3D structure of their vertebrate orthologs ${ }^{27,28}$. We reason that small differences between the respective orthologs' drug-binding pockets may facilitate the development of nematodeselective kinase inhibitors.

Second, inhibitors against a vast array of kinases have been developed (reviewed $\mathrm{in}^{29}$ ). Indeed, vertebrate kinases are the second most targeted class of proteins for drug development after the G-protein coupled receptors ${ }^{30}$. Currently, there are $52 \mathrm{FDA}$ approved small molecule inhibitors targeting protein kinases ${ }^{31}$ and over 200 are undergoing clinical trials for a variety of indications including cancer, inflammation, and autoimmune diseases ${ }^{32}$ (www.icoa.fr/pkidb/). Many more kinase inhibitors exist as pre-clinical research tools ${ }^{29}$. These include both ATP-competitive inhibitors and allosteric modulators. This well-established 'druggability' makes kinases attractive targets for anthelmintic exploration.

Finally, kinases are generally well-conserved. For example, the genome of the nematode Caenorhabditis elegans encodes 438 protein kinases and has close homologs of more than $80 \%$ of human kinases ${ }^{33}$. There is also a high degree of kinase conservation within the phylum Nematoda. For example, C. elegans has homologs of $95 \%$ of the kinases identified in the nematode parasite Haemonchus contortus ${ }^{34}$. Hence, drugs that target a particular vertebrate kinase may have utility against the respective ortholog in nematodes, and this kinase may be found broadly across the phylum.

Despite the conservation of kinases, there are sequence differences that distinguish nematode orthologs from their vertebrate counterparts. For example, the pairwise sequence similarity between homologous kinases of $H$. contortus and its sheep host Ovis aries is considerably lower ( $25 \%$ identity) than between $H$. contortus and C. elegans (35\% identity) ${ }^{34}$. A similar trend is found when comparing sequence similarity of orthologous kinases from the human nematode parasite Brugia malayi to that of $C$. elegans versus that of its human host system $^{21}$. These structural differences may be exploited to modify existing inhibitors of vertebrate kinases to derive nematode-selective inhibitors.

Here, we have identified candidate anthelmintic targets by exploiting the pharmacopeia of vertebrate kinase inhibitors. Our strategy consisted of three steps. First, we screened existing vertebrate kinase inhibitors for the ability to induce robust phenotypes in C. elegans. We focused on those inhibitors that yield a phenotype that mimics the loss-of-function of the respective orthologous kinase in the worm. C. elegans kinases that satisfy this criterium are considered, at least preliminarily, as 'druggable. Second, for these druggable C. elegans kinases, we inspected whether the inhibitor binding site diverges from vertebrates in relevant residues using homology modelling. Third, for those C. elegans kinases that satisfy the first two criteria, we asked whether C. elegans residues that diverge from mammals are conserved within the phylum Nematoda. With this subset of kinases, it may be possible to modify the structure of the respective vertebrate kinase inhibitors to confer nematode-selectivity. Finally, we used chemical genetic techniques and in vitro kinase activity assays to further investigate three of these druggable kinases that may be exploited to develop novel anthelmintics.

\section{Results}

A screen of vertebrate kinase inhibitors reveals 17 druggable nematode kinases. Towards identifying kinases that may serve as anthelmintic targets, we first screened 2040 vertebrate kinase inhibitors across four chemical libraries to identify those that disrupt the lifecycle of C. elegans (Fig. 1; Supplementary File S1). 1160 of these molecules are kinase inhibitors that are used as either research tools or medicines and come from the DiscoveryProbe Kinase Inhibitor Library (APExBIO), the Ontario Institute for Cancer Research (OICR) Kinase Inhibitor Library and a subset of the Library of Pharmacologically Active Compounds (LOPAC, Sigma-Aldrich). We refer to these molecules as 'commercial' inhibitors. The remaining 880 molecules are from GlaxoSmithKline's (GSK) Published Kinase Inhibitor Sets 1 and 2 (PKIS1 and PKIS2) ${ }^{35-37}$. GSK publicly released the PKIS library to foster basic research and academic drug discovery ${ }^{36}$. The core scaffolds in the PKIS were chosen based on previously published campaigns that targeted specific protein kinases, and these molecules were further screened against a panel of over 200 human kinases to assess selectivity ${ }^{35-37}$. Hence, a preliminary assessment of the in vitro inhibitory profiles of the PKIS molecules is known and span a range of kinase selectivity profiles from narrow to broad spectrum. Many of the PKIS scaffolds are represented by multiple analogs within the library. Consequently, hits from the PKIS library can provide insight on structure-activity relationships (SAR). The four libraries that we screened contained a total of 1716 unique inhibitor structures.

To identify druggable nematode kinases, we screened these kinase inhibitors for their ability to disrupt the viability, development and/or fecundity of C. elegans. We examined growth of the C. elegans culture after six days of co-incubation with the small molecules. We visually inspected the cultures to identify those compounds that induced any obvious phenotypes, including lethality (Let), larval arrest (Lva), sterility (Ste) and/or embryonic lethality (Emb) (Fig. 1A). We screened each compound at a final concentration of $10 \mu \mathrm{M}$ (OICR) or $60 \mu \mathrm{M}$ (APExBIO, LOPAC, PKIS), which were technically convenient concentrations at which most molecules remain 


\section{A}

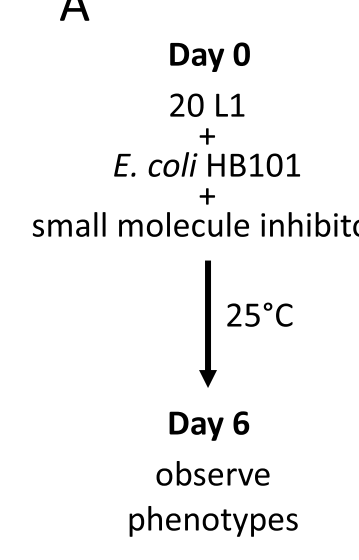

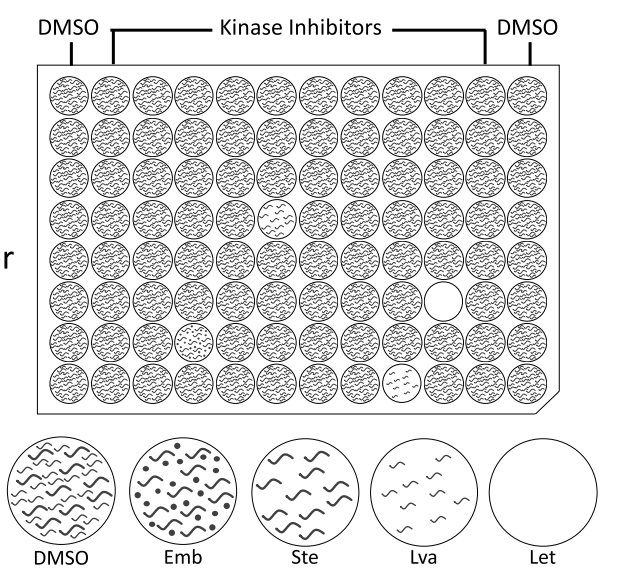

B 2040 vertebrate kinase inhibitors LOPAC (84 KIs)

APExBIO DiscoveryProbe KIL (436) OICR KIL (640) PKIS library (880)

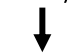

191 screen hits induce Emb/Ste/Lva/Let phenotypes $\downarrow$

130 vertebrate kinases targeted by screen hit molecules

$\downarrow$

114 have $C$. elegans orthologs

Genetic LOF phenocopies inhibitor exposure for 37 unique $C$. elegans kinases 17 of these $C$. elegans kinases targets
by $>1$ unique inhibitor within scree
17 candidate 'druggable'
nematode kinases (Table 1)

Figure 1. A screen of vertebrate kinase inhibitors reveals 17 druggable nematode kinases. (A) Schematic of small molecule screening methodology. (B) The pipeline used to identify candidate nematode kinase targets for structure analysis.

in solution. These concentrations may be considered high for in vitro and cell-based screening platforms, but are suitable for whole-animal C. elegans screens due to the nematode's robust xenobiotic defenses ${ }^{38,39}$. Using the criteria defined in the methods section, we identified 191 hits comprising 170 distinct inhibitors that reportedly target 130 vertebrate kinases that either kill, sterilize, or arrest the growth of C. elegans (Fig. 1B; Supplementary File S1A; Supplementary Table 1).

We next investigated whether these 191 vertebrate kinase inhibitors likely inhibit the orthologous C. elegans target or yield phenotype because of off-target effects. To do so, we asked whether the terminal phenotypes induced by a given kinase inhibitor phenocopied the genetic loss-of-function or RNAi phenotypes reported for the orthologous worm target on WormBase (WormBase web site, http://www.wormbase.org, release WS275, date $04-03-2020)^{40}$. We found that inhibitors induce phenotypes consistent with the genetic loss-of-function for 37 unique C. elegans kinases. We recognize that off-target phenotypes may be coincident with the anticipated phenotype and may confound interpretation at this point in the pipeline. However, downstream analyses (see below) further probe the relationship between the bioactive inhibitors and the anticipated targets.

To generate a higher-confidence list of druggable C. elegans kinases, we filtered the list of 37 kinases for those whose vertebrate orthologs were targeted by multiple structurally-distinct inhibitors that yielded the expected C. elegans loss-of-function phenotypes. In this way, we identified 17 higher-confidence druggable essential nematode kinase targets (Fig. 1B; Table 1). Regardless of the ultimate anthelmintic utility of these 17 druggable kinases, the respective inhibitors may be useful chemical tools for the community, allowing for the temporal control of kinase activity and simplifying the investigation of these essential cellular components in C. elegans.

Three kinases are candidate targets for anthelmintic development. We investigated whether any of the 17 higher-confidence kinase targets exhibit primary sequence divergence between nematodes and vertebrates within the drug-binding pocket. This divergence could then be exploited to develop nematode-selective inhibitors. To do this, we first used the SWISS-MODEL pipeline to generate protein homology models, mapping the C. elegans primary sequence onto the experimentally derived 3D crystal structure of the respective vertebrate kinase ortholog ${ }^{41}$. These homology models were visualized using PyMOL (The PyMOL Molecular Graphics System, Version 2.1.1, Schrödinger, LLC).

To test the validity of a homology modeling approach, we first created a homology model of the C. elegans LET-23 kinase domain based on the solved structure of the orthologous human EGFR kinase domain (Protein Data Bank (PDB) structure 2ITX ${ }^{42}$ ) (Supplementary Fig. 1A). We then compared the LET-23 homology model to the experimentally derived crystal structure of the LET-23 kinase domain (PDB: 5WNO ${ }^{43}$; Supplementary Fig. 1B). We found that the side chains of the residues that line the ATP-binding (and drug-binding) pocket are similarly oriented towards the active site in both models. This analysis supports the utility of the homology modeling approach to compare presumptive drug binding pockets of nematode kinases to orthologous vertebrate structures.

For each of the 17 druggable nematode kinases, we examined whether any residues that line the drug-binding pocket of the C. elegans kinase diverge from their respective vertebrate ortholog. Particular attention was paid to those residues that reside within $5 \AA$ of the bound inhibitor (Supplementary Fig. 2). To determine whether the pocket-lining residues that diverge in C. elegans also diverge within diverse parasitic nematode species, we generated and analyzed multiple sequence alignments. This pipeline revealed three potential anthelmintic targets 


\begin{tabular}{|c|c|c|c|c|}
\hline $\begin{array}{l}\text { Vertebrate } \\
\text { target }\end{array}$ & $\begin{array}{l}\text { C. elegans } \\
\text { Ortholog }\end{array}$ & \# Hits & Exemplar inhibitor $^{\mathrm{a}}$ & $\begin{array}{l}\text { Relevant } \\
\text { phenotypes }^{b}\end{array}$ \\
\hline EGFR & \multirow{4}{*}{ LET-23 } & 18 & \multirow{4}{*}{$\begin{array}{l}\text { Tyrphostin 23* } \\
\text { GSK306886A } \\
\text { GW583373A }\end{array}$} & \multirow{4}{*}{ Let Ste Rbs } \\
\hline ERBB2 & & 6 & & \\
\hline ERBB3 & & 1 & & \\
\hline ERBB4 & & 9 & & \\
\hline MAP2K1 & \multirow{2}{*}{ MEK-2 } & 13 & \multirow{2}{*}{ Trametinib $^{*}$} & \multirow{2}{*}{ Emb Lva Let Ste Rbs } \\
\hline MAP2K2 & & 13 & & \\
\hline \multirow{2}{*}{ PLK1 } & PLK-1 & \multirow{2}{*}{9} & \multirow{2}{*}{$\begin{array}{l}\text { MLN0905* } \\
\text { GSK580432A }\end{array}$} & Emb Let Ste Rbs \\
\hline & PLK-2 & & & Emb Ste Rbs \\
\hline BRAF & \multirow{3}{*}{ LIN-45 } & 14 & \multirow{3}{*}{ Dabrafenib(GSK2118436)* } & \multirow{3}{*}{ Emb Let Ste } \\
\hline RAF1 & & 12 & & \\
\hline ARAF & & 1 & & \\
\hline AURKB & \multirow{3}{*}{ AIR-2 } & 10 & \multirow{3}{*}{$\begin{array}{l}\text { SNS-314 Mesylate } \\
\text { AMG- } 900^{*}\end{array}$} & \multirow{3}{*}{ Emb Let Ste Rbs } \\
\hline AURKA & & 6 & & \\
\hline AURKC & & 4 & & \\
\hline MTOR & LET-363 & 5 & Torin $1^{*}$ & Emb Lva Let Ste \\
\hline CDK1 & CDK-1 & 4 & Cdk1/2 Inhibitor III* & Emb Ste \\
\hline MAPK11 & \multirow{2}{*}{ PMK-1 } & 4 & \multirow{2}{*}{ SB-245392 } & \multirow{2}{*}{ Let or Ste } \\
\hline MAPK14 & & 3 & & \\
\hline SRC & \multirow{3}{*}{ SRC-1 } & 4 & \multirow{3}{*}{$\mathrm{PP} 2^{*}$} & \multirow{3}{*}{ Emb Let } \\
\hline LCK & & 2 & & \\
\hline FYN & & 1 & & \\
\hline IGF1R & \multirow{3}{*}{ DAF-2 } & 3 & \multirow{3}{*}{ GSK1838705A* } & \multirow{3}{*}{ Let Ste Rbs } \\
\hline INSR & & 2 & & \\
\hline INSRR & & 1 & & \\
\hline STK10 & GCK-4 & 3 & & Lva Let Rbs \\
\hline CSNK1E & \multirow{2}{*}{ KIN-20 } & 3 & & \multirow{2}{*}{ Emb Let Ste Rbs } \\
\hline CSNK1D & & 2 & & \\
\hline GSK3A & \multirow{2}{*}{ GSK-3 } & 2 & \multirow{2}{*}{ AZD2858* } & \multirow{2}{*}{ Emb Let Ste } \\
\hline GSK3B & & 2 & & \\
\hline BUB1 & BUB-1 & 2 & GSK350559A & Emb Lva Let Ste \\
\hline CSNK2A1 & \multirow{2}{*}{ KIN-3 } & 2 & & \\
\hline CSNK2A2 & & 1 & GW85/175X & Emb Lva Ste \\
\hline CSNK1A1 & KIN-19 & 2 & & Emb Ste \\
\hline
\end{tabular}

Table 1. Druggable essential nematode kinase targets. ${ }^{a}$ Commercially available inhibitors indicated with an asterisk $\left({ }^{*}\right) .{ }^{b}$ Embryonic Lethal (Emb); Larval Arrest (Lva); Lethal (Let); Sterile (Ste); Reduced Brood Size (Rbs).

that include EGFR (LET-23 in C. elegans), the MAP kinase kinase MEK1 (MEK-2 in C. elegans), and polo-like kinase PLK1 (PLK-1 in C. elegans) (Fig. 2). Structural modeling of these three kinase domains from the human parasitic nematode $B$. malayi confirmed that the divergent residues line the prospective drug binding pockets and provide an opportunity to develop nematode-selective kinase inhibitors (Supplementary Fig. 2).

LET-23 divergence may interfere with its ability to interact with EGFR inhibitors. Our pipeline revealed LET-23, the C. elegans EGFR ortholog, as a candidate anthelmintic target. LET-23 and its conserved signaling pathway (Fig. 3) regulates a wide variety of developmental processes including vulval induction and excretory duct cell formation and is one of the best understood receptor tyrosine kinases in C. elegans ${ }^{23,24,44,45}$. LET-23, which is expressed in the presumptive vulval epithelial cells, responds to LIN-3/EGF that is secreted by the nearby anchor cell to coordinate the development of the vulva tissue ${ }^{46}$. Too little signaling through LET-23 renders cells incapable of developing vulva tissue, resulting in what is known as a vulvaless (Vul) phenotype ${ }^{47}$. LET-23 also plays a Ras-independent role in promoting ovulation through the $\mathrm{PLC} \gamma-\mathrm{IP}_{3}$ pathway, resulting in a sterile (Ste) phenotype upon loss of LET-23 function ${ }^{24}$. Weak let-23 mutants such as let-23(sy1) are vulvaless but not sterile. Consequently, let-23(sy1) adults are filled with internally hatched embryos that destroy their hermaphrodite mothers, which is a phenotype known as bag-of-worms (Bag). When LET-23 pathway signalling is overactive, too many cells develop into primary vulval tissue, creating a multivulva (Muv) phenotype ${ }^{48}$. LIN-3/ LET-23 signaling via Ras-ERK also plays an integral role in excretory duct cell fate specification, morphogenesis and differentiation ${ }^{49,50}$. Proper development of the excretory duct cell is required for osmoregulation ${ }^{51,52}$. Homozygous null let-23 animals are therefore larval lethal and die with a distinctive rod-like, fluid-filled appearance due to the loss of the excretory duct cell ${ }^{49}$. 
A EGFR/LET-23 interaction with Lapatinib

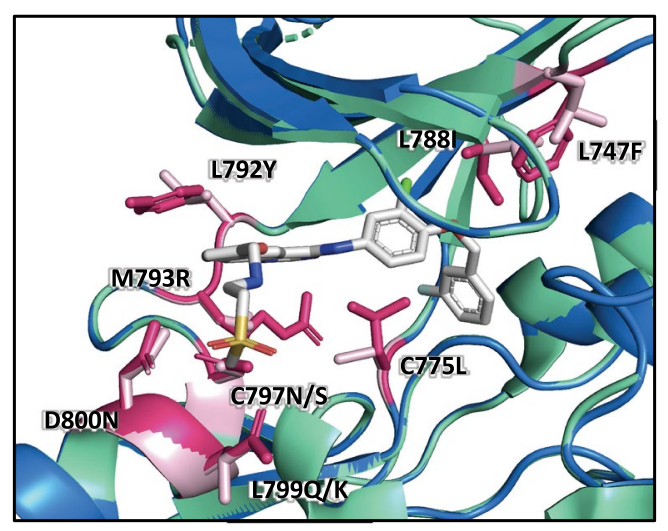

B MEK1/MEK-2 interaction with BI-847325

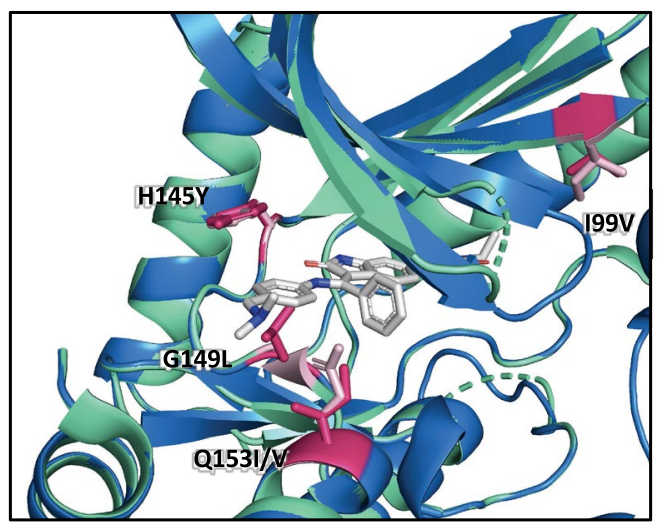

C PLK1/PLK-1 interaction with BI-2536

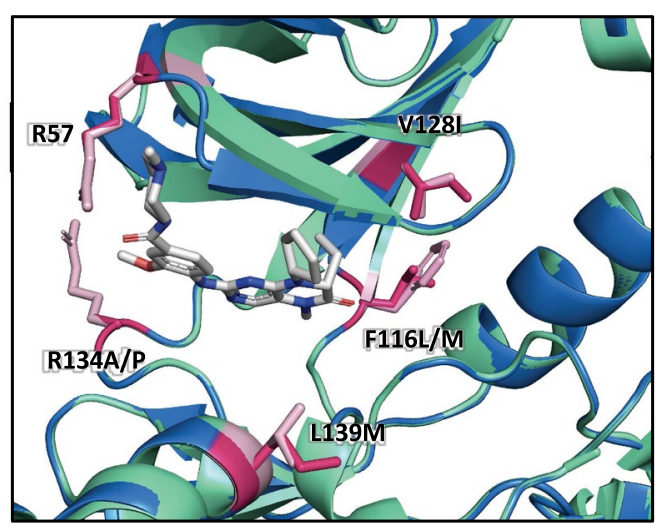

Human crystal structure Human residue

C. elegans homology model C. elegans residue

$A^{\prime}$

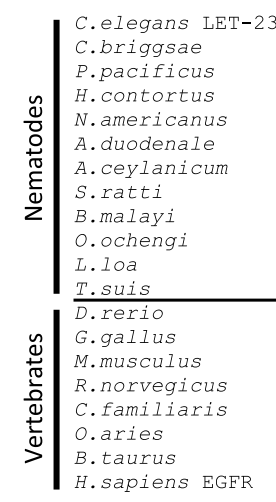

$\hat{\imath}$

ヘ⿱⺊口冋

$\left.1^{80} 191^{2}\right)^{3}+949_{9}^{0}$

IKVFQTHDNLLKI I GFCMHDDGLKIVTI YRPLGNLQNFLKL IKIFQVEEHLLKI I GFC I HDDGLKIVT I YRPLGDLHSFLQL IKVVRD HENLLRIVGVCLSGDDLQLVTLLRPLGNLREFLQK IKLVKG HEHLLRLVGICLHEEG IQLVTLLRPLGNLLNFLKK IKLVKGHEHLLRLVGICLHEEGTOI-

IKLVKGHEHLLRLVGICLHEEGIOLVTLLRPLGNLLNFLKK 作 IKLVKG EEHLLRLVGICLHEEG IQLVTLLRP LGNLLNFLKK I KVMKA AKHLHP I IGICFC-DTVQIVTLLRPLGSLLHFLKK IKVVHD EE HLIRLVGVCLS-DGMQ IVTPMRPLGSLKNYLKK IKVVND EEHLLRLVGVCLS-NGMQIVTPMRPLGSLKNYLKK IKVVHD HEHLLRLVGVCLS-DGMQIVTPMR PLGSLKNYLKK IKVLHE HPHLVQLIGVCVG-OOMMLVT PLMPLGNLIDYVON

IKVLRE HPHVCRLLGICLT-STVOLITQLMPYGCLLDYVRE

IKELRE NPHVCRLLGICLT-STVQLITQLMPYGCLLDYIRE IKELRE NPHVCRLLGICLT-STVQL ITQLMPYGCLLDY IRE IKELRE NPHVCRLLG ICLT-STVQI I TQLMPYGCLLDYVRE
IKELRENPHVCRLLGICLT-STVQLITQLMPYGCLLDYVRE IKELRE NPHVCRLLGICLT-STVQLITQLMPFGCLLDYVRE IKELRE NPHVCRLLGICLT-STVQLITOLMPFGCLLDYVRE IKELRE NPHVCRLLGICLT-STVQLITOLMPFGCLLDYVRE IKELRENPHVCRLLGICLT-STVQLITQLMP FGCLLDYVRE $*$ $* \quad * * *$

B'

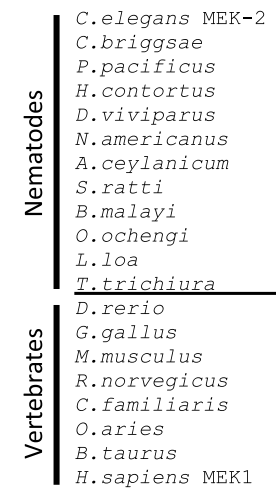

के

MARKLVHLEIKPSDIS ICMEYMDGLSLDIVLKKVGRLPEKF MARKLVHLEIKPS DIS ICMEYMDGLS LDIVLKKVGRLPEKF MARKLVHLEVKPSD IS I CME YMDGRS LDLVLKKVTRLQENF MARKLVHLEVKPSD IS ICMEYMDGLS LDIVMKKVGRIAEKF MARKLVHLEVKPSD IS I CMEYMDGL S LD IVMKKVGRI PEKF MARKLVHLEVKPSD IS I CMEYMDGLS LDIVMKKVGRI PEKE MARKLVHLEVKPS D IS I CMEYMDGLS LDIVMKKVGRI PEKF MAAKMVHLEVKPTD IS I CME YMD GLS LDI IVQRS GR I HEN I MARKL VHL EVKPSD ISICMFYMDGLSLDVVLKKVGRL KESR MARK MARKLVHLEVKPSDIS ICMEYMDGLS LDVVLKKVGKLKESR MARKLVHLEVKPSD IS ICMEYMDGLS LDVVLKKVGKLKESR
MARKLIHLEVKPSE ISMCMEYMDGLS LDIVLKKTGRFAEOI MARKLIHLE I KPAE IS ICMENMDGGS LDQCLKKAGKI PEQI MARKLIHLE I KPAE IS I CMEHMDGGS LDQVLKKAGR I PEQI MARKLIHLE I KPAF IS I CME HMDGGS LDQVLKKAGR I PEQI MARKLIHLEIKPAE IS I CMEHMDGGSLDOVLKKAGRIPEOI MARKIIHLEIKPA TSICMETM MARKIIHLEIKPA ISICMEHMGGSIDQVIKKAGRIPEQI MARKLIHLEIKPATISICMEHMDGGSLDQVLKKAGRIPEQI MARKLIHLE I KPAE IS I CMEHMDGGS LDQVLKKAGRI PEQI MARKLIHLEIKPAEI I ICMEHMDGGS LDQVLKKAGRI PEQI * * *

\section{$\mathbf{C}^{\prime}$}

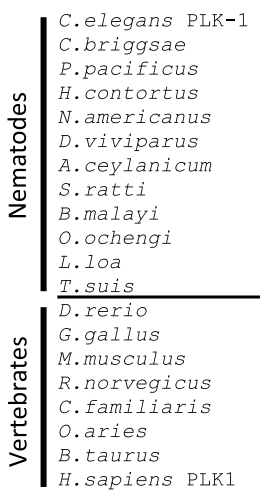

\section{$\hat{n}$} YEKGRFLGK VIVKLFHFFEDSLNVYITLELCARRS LMELHK YARGRF LGK HVVKI I DYFEDDDNVYV I LE LCARRS LMELHK Y IRGKF LGK HVVQI YNFFEDHDNVY IT LELCARRS LME LHK Y IRGKF LGK HVVQLYSFFE DVDNVYITLELCARRSLMELHK Y IRGKF LGK HVVQL YNFFE DNENVY IT LELCARRS LMELHK YIRGKFLGK HVVOLYSFFEDADNVYITLFICRRRSI YIRGKF CX RSIMELHK YKKGRF LGK I IVRMDGFFEDTDNVY I LLELCPRRS LMELHK YKKGRFLGK IIVRMDGFFEDADNVY I LLELCPRRS LMELHK YKKGRFLGK IIVRMDGFFEDP DNVY I LLELCPRRS LMELHK YIKGKFLGK VIVKFYRHFEDDENVYILLELCCRRS LMELHK YVRGRFICK HVVGEOSEFEDGDFVYVVI TLCRRSIIFIHK YVRGRF YVRGR LGK IVVGE YVRGRFLGK HVVGFHGFFEDSDFVFVVLELCRRRSLLELHK YLRGRF LGK IIVGFHGFFE DSDFVFVVLELCRRRS LLELHK YLRGRF LGK HVVGFHGFFE DNDFVFVVLELCRRRSLLELHK YLRGRFLGK HVVGFHGFFEDNDFVFVVLELCRRRSLLELHK YVRGRFLGK/VVVGFHGFFEDNDFVFVVLELCRRRSLLELHK
YEKGRFLGKNIVKLFNFFEDNLNVYITLELCARRSLMELHK

Figure 2. Structure analysis identifies LET-23, MEK-2 and PLK-1 as candidate targets for anthelmintic development. C. elegans homology models (blue) for LET-23, MEK-2 and PLK-1 aligned with human crystal structure (green) for EGFR (PDB: 1XKK) ${ }^{86}$, MEK1 (PDB: 5EYM) ${ }^{59}$ and PLK1 (PDB: 2RKU) ${ }^{64}$ are shown in (A-C) respectively. Key divergent residues proximal to the inhibitor binding sites are highlighted in dark pink (C. elegans residue) and light pink (human residue) within the structure diagrams (A-C). Residues are labeled according to their position in the human kinase, with the first letter indicating the identity of the vertebrate residue and the latter indicating the identity of the nematode residue(s). The conservation of these residues among free-living nematodes, parasitic nematodes and vertebrates is displayed in the corresponding sequence alignments $\left(\mathbf{A}^{\prime}-\mathbf{C}^{\prime}\right)$. The vertical line indicates a discontinuous break in the sequence. The horizontal line indicates the separation between the nematode and vertebrate sequences. Yellow residues in the sequence alignments highlight those nematode residues that differ in identity from both vertebrate and C. elegans sequence at the location of these divergent residues of interest. Residues that have distinct physicochemical properties between vertebrates and nematodes are indicated with an asterisk below the alignment. 
A
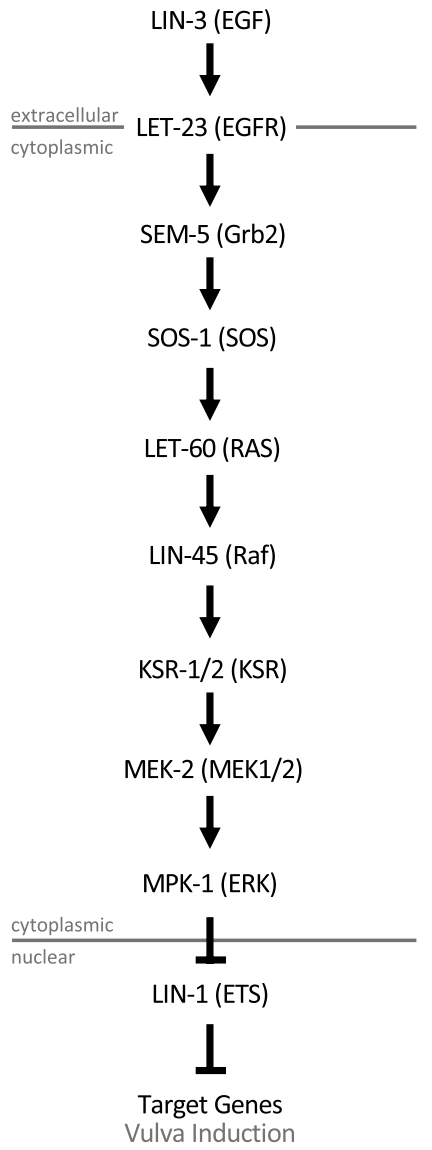

B
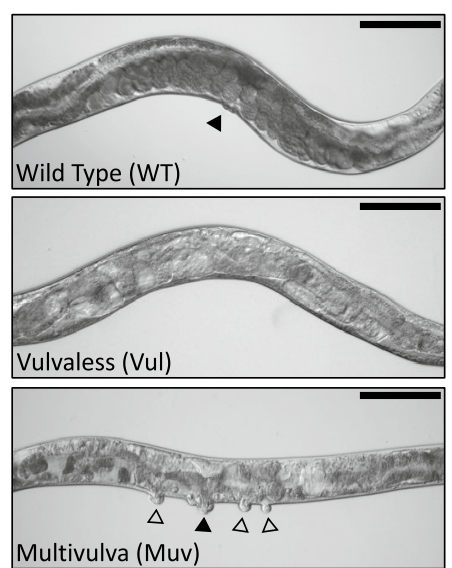

Figure 3. The conserved C. elegans EGFR/Ras/MAPK pathway controls vulval induction signaling. (A) A schematic of the conserved EGFR signaling pathway in nematodes. The vertebrate orthologs for each pathway component are shown in brackets. Changes in signaling levels through this pathway result in the Vulvaless (Vul) and Multivulva (Muv) vulva induction phenotypes shown in (B). Filled arrows indicate primary functional vulva, clear arrows indicate ectopic vulval protrusions. Scale bar $0.1 \mathrm{~mm}$.

Our collection of kinase inhibitors include 66 distinct commercial inhibitors of EGFR or paralogous ERBBs (Supplementary File S2A). Of these, only two (Tyrphostin AG879 and Tyrphostin 23) induce robust phenotypes in C. elegans. One possibility for why so few of these inhibitors induce let-23 phenotypes is that they may have unfavorable ADME (adsorption, distribution, metabolism and excretion) properties in nematodes and fail to reach target. We tested this possibility by asking whether one of these molecules, gefitinib, could induce let-23-like phenotypes in a humanized strain of C. elegans in which the kinase domain of LET-23 is replaced by that from human EGFR. This strain was kind gift from Dr. Jaegal Shim ${ }^{53}$. Previous work demonstrated that the humanized LET-23 could rescue the vulvaless phenotype of the let-23(sy1) mutant, which we confirmed (Fig. 4). Gefitinib failed to induce a vulvaless phenotype in wild type animals at the $60 \mu \mathrm{M}$ concentration tested, but did induce 


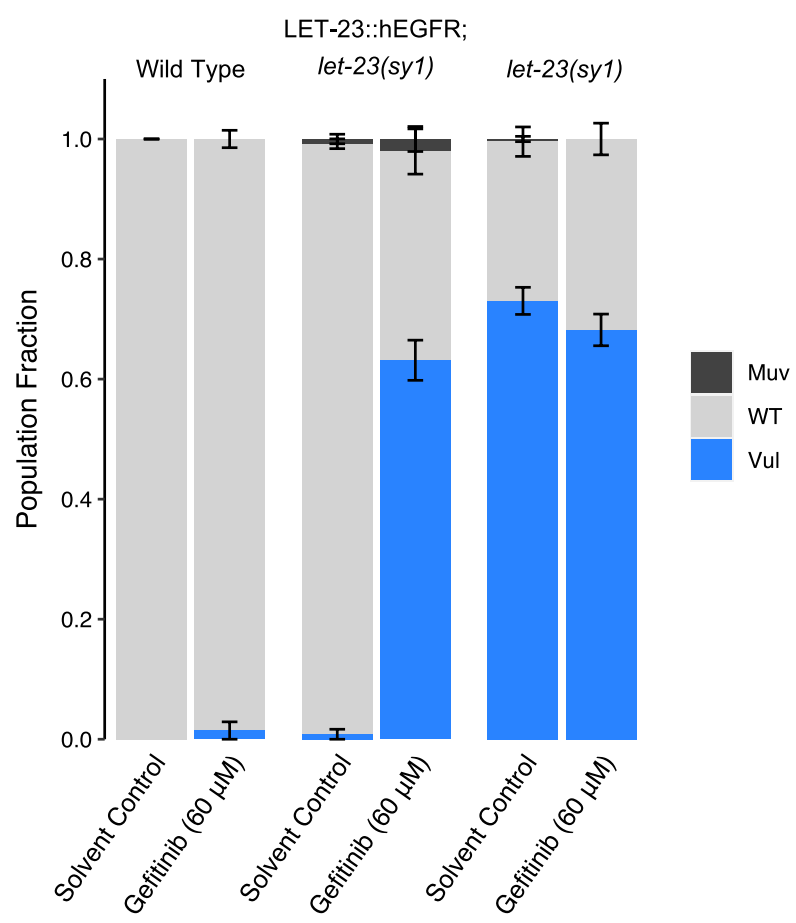

Figure 4. Structural divergence in EGFR/LET-23 impacts response to EGFR inhibitor gefitinib. The Vulvaless phenotype observed in the let-23(sy1) mutant is rescued by the expression of a chimeric protein whereby the kinase domain of LET-23 is replaced with human EGFR (LET-23::hEGFR). Gefitinib induces a vulvaless phenotype in the humanized strain, but not in wild type animals. DMSO is used as the solvent control. Error bars indicate SEM.

a vulvaless phenotype in the humanized strain (Fig. 4). This data indicates that ADME barriers are not solely responsible for gefitinib, and perhaps other EGFR/ERBB inhibitors, failing to inhibit worm LET-23.

A second possibility for why so few EGFR/ERBB inhibitors disrupt LET-23 is that there may be structural differences within the drug-binding pocket of LET-23 that hinders interaction with EGFR/ERBB inhibitors. We investigated this possibility by comparing the structure of EGFR's drug binding site to that of C. elegans LET-23 (as described above-see Supplementary Fig. 2A). Overall, there is $44 \%$ identity in sequence in the kinase domain between LET-23 and human EGFR. Within the drug-binding pockets of the human and C. elegans structures, we found 10 obvious amino acid differences (Supplementary Fig. 2A), eight of which also vary between vertebrate hosts and parasitic species (Fig. 2A'). Five of these eight residues have distinct physicochemical properties from the vertebrate residues and could account for differential compound binding (Fig. 2A'). We conclude that the structural differences within the presumptive drug-binding pocket of LET-23 likely accounts for the inability of the EGFR/ERBB inhibitors to induce let-23 phenotypes. It is these structural differences that may allow for the rational design of nematode-selective EGFR inhibitors.

Two scaffolds for the development of nematode-selective LET-23 inhibitors. We identified 20 PKIS inhibitors that induce let-23-like hypomorphic phenotypes in C. elegans (Fig. 5A) and show some degree of selectivity for EGFR/ERBB inhibition (Supplementary File S2B) ${ }^{35-37}$. These compounds fall within three related core scaffolds: the anilino thienopyrimidines (ATOPs); the $\underline{4}$-anilino quinazolines (4AQs); and a $4 \mathrm{AQ}$ derivative scaffold called the quinazoline benzimidazoles (QBIs) (Fig. 5B). The quantities of these compounds available to us did not allow for extensive follow up experiments. However, we were able to test at least one exemplar from each scaffold (GSK306886A (an ATOP), GW583373A (a 4AQ), GW576484X (a 4AQ) and GW272142A (a QBI)) in dose-response analyses in C. elegans. The ATOP GSK306886A and 4AQ GW583373A exhibited additional let-23-like phenotypes that include Bag and sterility in the dose-response analysis (Fig. 5B). We also probed the ability of these four structures to disrupt LET-23 kinase activity in vitro. We observe inhibition of LET-23 kinase activity for both the ATOP and 4AQ molecules (Fig. 5C). The relative insolubility of the molecules in the kinase assay buffer prevented us from ascertaining accurate IC50s of the inhibitors (see methods for details). Regardless, the data suggest that the ATOP and 4AQ scaffolds likely inhibit LET-23 function in vivo.

By analysing the bioactivity of $4 \mathrm{AQ}$-based structures in C. elegans, we were able to complete a small SAR analysis (Supplementary Fig. 3). We found several substructural features that may improve activity in C. elegans, including a thiomorpholine 1-oxide ring structure in the $\mathrm{R}_{1}$ position, a furan as opposed to a thiazole as the $\mathrm{X}_{1-3}$ substituted ring and a halogen in the $\mathrm{R}_{5}$ position. These features are embodied in GW576609B, which could serve as a key scaffold on which to base the rational design of a nematode-selective EGFR inhibitor. An insufficient number of QBI and ATOP analogs precluded us from performing a similar SAR analysis on these scaffolds. 
A

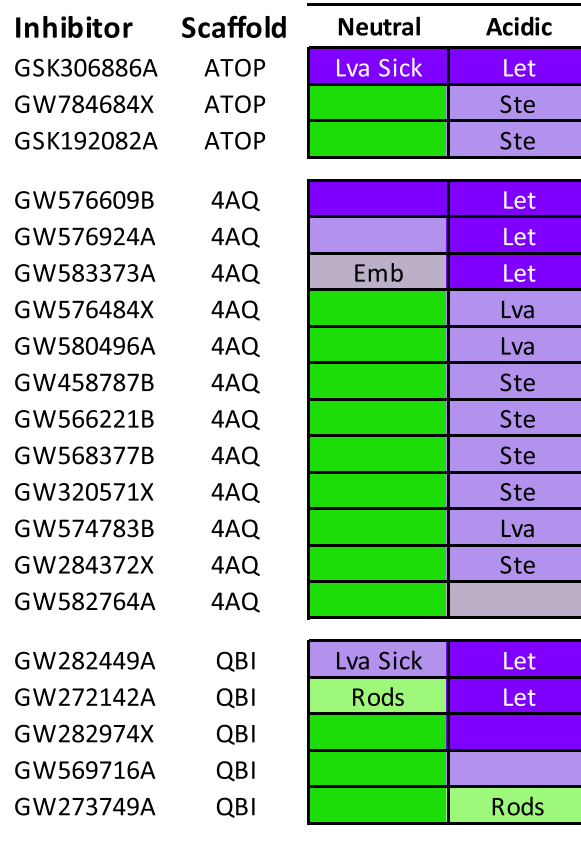

\begin{tabular}{|l|l|l|l|l|}
\hline$>50 \mathrm{nb}$ & $>50$ & $26-50$ & $11-25$ & $0-10$ \\
\hline
\end{tabular}

\# living worms per well
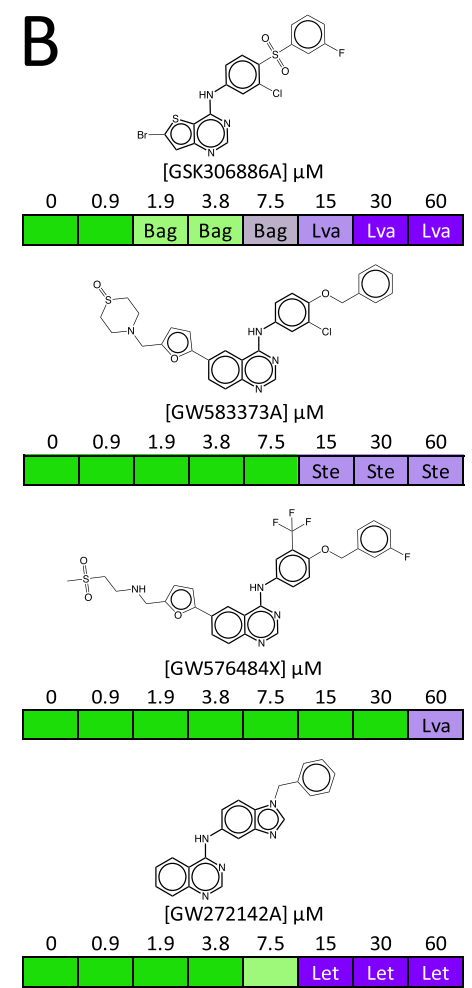

\section{C}

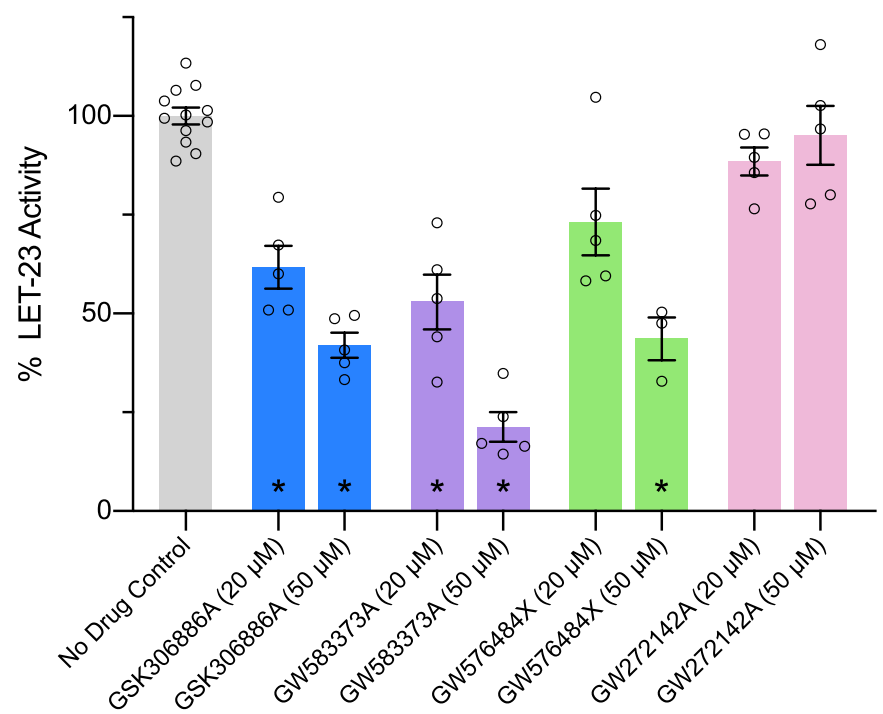

Figure 5. Three scaffolds induce LET-23 loss-of-function phenotypes in C. elegans. (A) The structurally related anilino thienopyrimidine (ATOP), 4-anilino quinazoline (4AQ), and quinazoline benzimidazole (QBI) scaffolds induce LET-23 loss-of-function phenotypes in our C. elegans chemical screens. (B) Exemplar worm-active structures from each scaffold are shown along with the associated dose-response in wild type C. elegans. These dose-response analyses reveal phenotypes relevant to EGFR/MAPK pathway inhibition including sterility (Ste) and the bag-of-worms phenotype (Bag). Additional phenotypes including lethality (Let) and larval arrest (Lva) are shown and the resulting population growth defects are indicated by the colour coded scale (nb, no bacteria remaining in the well). Dose-response analyses for GSK306886A and GW583373A were performed in neutral media, GW576484X and GW272142A in acidic media. (C) Four worm-active inhibitors were tested for their ability to inhibit $C$. elegans LET-23 kinase activity in vitro. LET-23 inhibition is expressed as a the percentage of total kinase activity observed in no drug controls averaged across 3-5 replicates for each inhibitor condition. The ATOP and 4AQ scaffold compounds tested were able to inhibit LET-23 kinase activity (Student's T-test; $\left.{ }^{*} p<0.01\right)$. Error bars indicate SEM. 


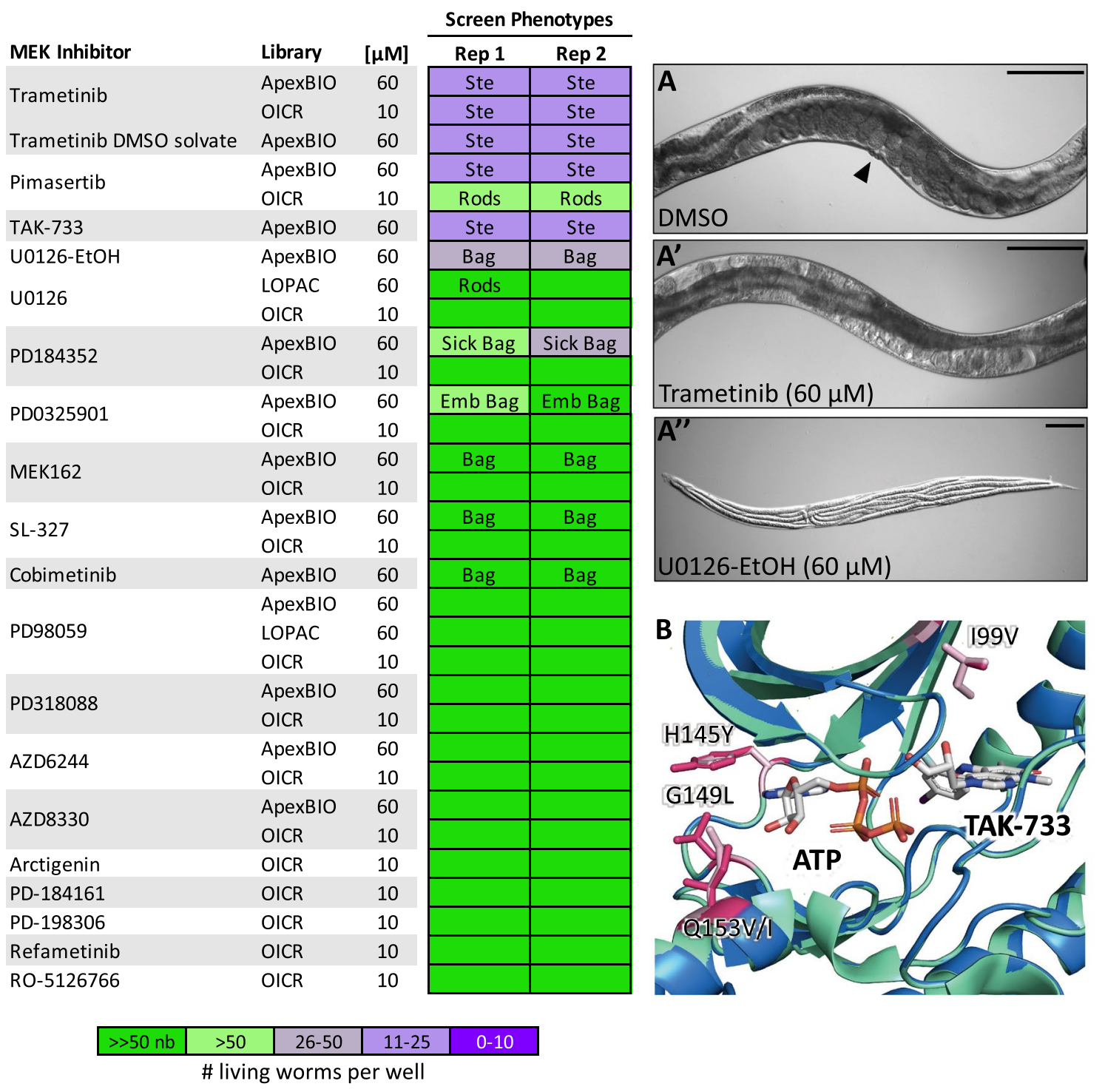

Figure 6. Allosteric MEK inhibitors induce sterility, embryonic lethality and vulva development phenotypes in C. elegans. 18 unique MEK inhibitors from the commercial libraries screened were included in our screen. These inhibitors were enriched for hits that induce the expected MEK-2 loss-of-function phenotypes including sterility (Ste) and the bag-of-worms phenotype (Bag), resulting from vulval induction defects preventing egg-laying (A-A", scale bar $0.1 \mathrm{~mm})$. The resulting population growth defects are indicated by the colour coded scale (nb, no bacteria remaining in the well). (B) The allosteric inhibitor binding site of C. elegans MEK-2 (in blue) is well conserved with that of vertebrate MEK1 (in green, seen co-crystalized with allosteric inhibitor TAK-733 (PBD: 3PPI). The allosteric site contains only one amino acid difference, highlighted in pink (I99V).

MEK-2 is a Druggable Kinase with Conserved Divergence Among Nematodes. Our pipeline revealed worm MEK-2 as a candidate anthelmintic target. MEK-2 is a MAP kinase kinase ortholog of mamma-

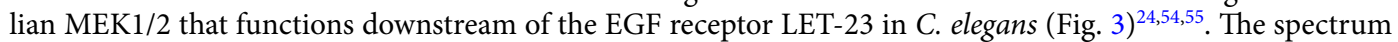
of worm mek-2 loss-of-function phenotypes is similar to that of let-23 mutants; mek-2 loss-of-function mutants die as rod-like sticks while weaker reduction-of-function mutants that escape lethality become sterile and/or vulvaless ${ }^{54}$.

The kinase domain of worm MEK-2 is $60 \%$ identical to vertebrate MEK1. Much of the ATP/drug binding pocket of nematode MEK-2 is identical to vertebrate MEK1. However, there are three divergent residues with distinct properties on the hinge that connect the $\mathrm{N}$ and $\mathrm{C}$ lobes (Fig. 2B, Supplementary Fig. 2B). The divergence is well-conserved within the phylum Nematoda (Fig. 2B'). Compounds that interact with MEK1/2 near the hinge may therefore have the potential to be modified for increased nematode selectivity over the vertebrate ortholog.

In our initial survey, we screened 18 unique commercial inhibitors of vertebrate MEK1/2 (Fig. 6). Nine of these inhibitors dramatically slowed population growth and/or induced mek-2-like hypomorphic phenotypes, including bag-of-worms, sterility, and embryonic lethality (Fig. 6A-A"). This activity suggested that MEK-2 is a druggable target. 
To validate worm MEK-2 as the target of these vertebrate MEK1/2 inhibitors in vivo we took a chemicalgenetic approach using three structurally distinct worm-active compounds, trametinib, pimasertib, and U0126EtOH. LET-60/RAS functions upstream of MEK-2 (Fig. 3) ${ }^{54,55}$. A gain-of-function allele of let-60(n1046) induces a partially penetrant multivulva (Muv) phenotype ${ }^{56}$. If a compound inhibits MEK-2, it should suppress the Muv phenotype of let-60(n1046). By contrast, the LIN-1 ETS transcription factor is a negative regulator of the EGFR/ MAPK pathway. A loss-of-function allele of lin-1(e1275) also results in a Muv phenotype. However, MEK-2 functions upstream of LIN-1 ${ }^{57}$. Hence, molecules that disrupt MEK-2 should not suppress the MUV phenotype of lin-1(e1275) animals. We found that all three MEK1/2 inhibitors suppressed the Muv phenotype of let-60(n1046) mutants at sub-micromolar concentrations and failed to suppress the Muv phenotype of lin-1(e1275) mutants up to the highest concentrations tested $(120 \mu \mathrm{M})($ Fig. $7 \mathrm{~A}-\mathrm{C})$. These data indicate that the MEK1/2 inhibitors likely act on their expected target in vivo and confirms that MEK-2 is a druggable target in nematodes.

Finally, we examined whether the three aforementioned allosteric MEK inhibitors induce mek-2-related lossof-function phenotypes in two additional free-living nematode species, C. briggsae and Pristionchus pacificus. We found that these inhibitors induce the expected phenotypes across all three nematode species in a concentrationdependent manner with trametinib being the most potent (Fig. $7 \mathrm{~A}^{\prime}-\mathrm{C}^{\prime}$ ). Together, these results show that MEK-2 may be an excellent anthelmintic target.

Divergence of the MEK-2 hinge may be exploited to develop nematode-selective inhibitors. The commercial MEK1/2 inhibitors that we screened are allosteric modulators that do not compete for the ATP binding pocket (Fig. 6). Instead, they bind near the base of the aC helix, which is distal to the divergent nematode sequence in the hinge region (Fig. 6B; PDB: $3 \mathrm{PP} 1^{58}$ ). It is therefore unlikely that these allosteric MEK inhibitors can be modified to be nematode-selective. By contrast, MEK1/2 ATP-competitive inhibitors may be far better scaffolds upon which to build nematode-selective analogs because the hinge that lines the ATP-binding pocket of nematode MEK-2 is divergent from that of vertebrate MEK1/2 (Fig. 6B).

We investigated whether the differences in nematode MEK1/2 structure are likely sufficient to confer phylum-selectivity. We first inspected the co-crystal structure of an ATP-competitive inhibitor of MEK1/2 called BI-847325 (PDB: 5EYM) ${ }^{59}$, which binds to the vertebrate MEK1/2 close to the hinge region (Fig. 2B). Inspection of the MEK-2 homology model reveals that the leucine of the nematode sequence (G149L) may sterically hinder BI-847325's terminal amine and prevent binding (Fig. 2B, Supplementary Fig. 2B). Of note, BI-847325 is one of the only established MEK1/2 selective inhibitors that competes for the ATP-binding pocket; the majority of MEK1/2 inhibitors are non-ATP competitive allosteric modulators ${ }^{60}$.

We tested the hypothesis that BI-847325 is unable to disrupt MEK-2 activity in vivo through a dose-response analysis. BI-847325 inhibits vulval induction at $120 \mu \mathrm{M}$ in wild type animals (Fig. 8A). If BI-847325 reduced vulval induction through the inhibition of MEK-2, then BI-847325 should suppress the multivulva (Muv) phenotype of the upstream RAS gain-of-function mutation (let-60(n1046)), but not the Muv phenotype of the down-stream ETS transcription factor loss-of-function mutation (lin-1(e1275)). Instead, we found that BI-847325 reduced vulval induction in both mutants, suggesting that BI-847325 can access target in the worm, but is likely modulating a target other than MEK-2 (Fig. 8A). BI-847325 is a dual inhibitor of vertebrate MEK and Aurora kinases ${ }^{59}$. Reduced vulva induction by BI- 847325 may be due to the inhibition of a C. elegans Aurora kinase. Indeed, loss of function of AIR-2 Aurora kinase ortholog in the worm results in vulvaless, embryonic lethality and sterility phenotypes in C. elegans ${ }^{61}$, which are all phenotypes induced by BI-847325 (Fig. 8A'). Furthermore, there is no sequence divergence in the drug binding pocket of worm AIR-2 (relative to human Aurora kinases) that would impede inhibitor binding (Supplementary Fig. 2E) ${ }^{59}$. We conclude that BI-847325 is bioavailable to the worm but based on our chemical-genetic and structural analyses, may be unable to effectively inhibit MEK-2. Hence, the divergence in the hinge region of worm MEK-2 may allow for the rational design of a nematode-selective ATP-competitive MEK inhibitor. The core scaffold of BI-847325 would be an appropriate starting point for such an effort.

PLK-1 is a High Priority Target for Anthelmintic Development. The C. elegans Polo-Like Kinase 1 ortholog PLK-1 regulates the meiotic cell cycle and embryonic polarity in the worm. C. elegans PLK-1 is $64 \%$ identical in the kinase domain to human PLK1. Loss-of-function mutations in plk-1 result in embryonic lethality and sterility phenotypes, along with a protruding vulva phenotype ${ }^{62,63}$. PLK1 inhibitors from three core scaffolds within the PKIS library induce phenotypes with a strong correlation to those observed upon genetic loss of plk-1. These include the benzimidazole N-thiophenes (BTs), the 2,4-dianilino pyrrolopyrimidines, and the 2,4-dianilino pyrimidines (Fig. 9A; Supplementary File S2D). The strong correlation between the established plk-1 mutant phenotypes and those caused by the PLK1 inhibitors across the three scaffolds gave us confidence in the potential of PLK-1 as a druggable target in the worm.

We modeled the protein structure of C. elegans PLK-1 against the crystal structure of human PLK1 (PDB:2RKU) ${ }^{64}$. Four residues in the ATP binding pocket differ between C. elegans PLK-1 and human PLK1 (Fig. 2C, Supplementary Fig. 2C). A multiple sequence alignment with additional nematode and vertebrate species shows that nematode residues differ at each of these locations, with a greater degree of conservation with the specific C. elegans amino acid among the parasitic Clade V nematodes (Fig. 2C'). A fifth residue of interest was identified (R57) that is conserved between C. elegans and vertebrates but differs in a number of the parasitic nematode sequences examined (Fig. $2 \mathrm{C}^{\prime}$ ).

We retested a selection of available PKIS compounds from the three core scaffolds of interest in C. elegans and two additional free-living nematode species, C. briggsae and P. pacificus (Fig. 9A). We found that the 2,4-dianilinopyrimidine GSK1520489 and the benzimidazole thiophene (BT) inhibitors GSK580432 and GSK479719 induced relevant embryonic lethality and/or sterility phenotypes across all species tested. To test whether these 

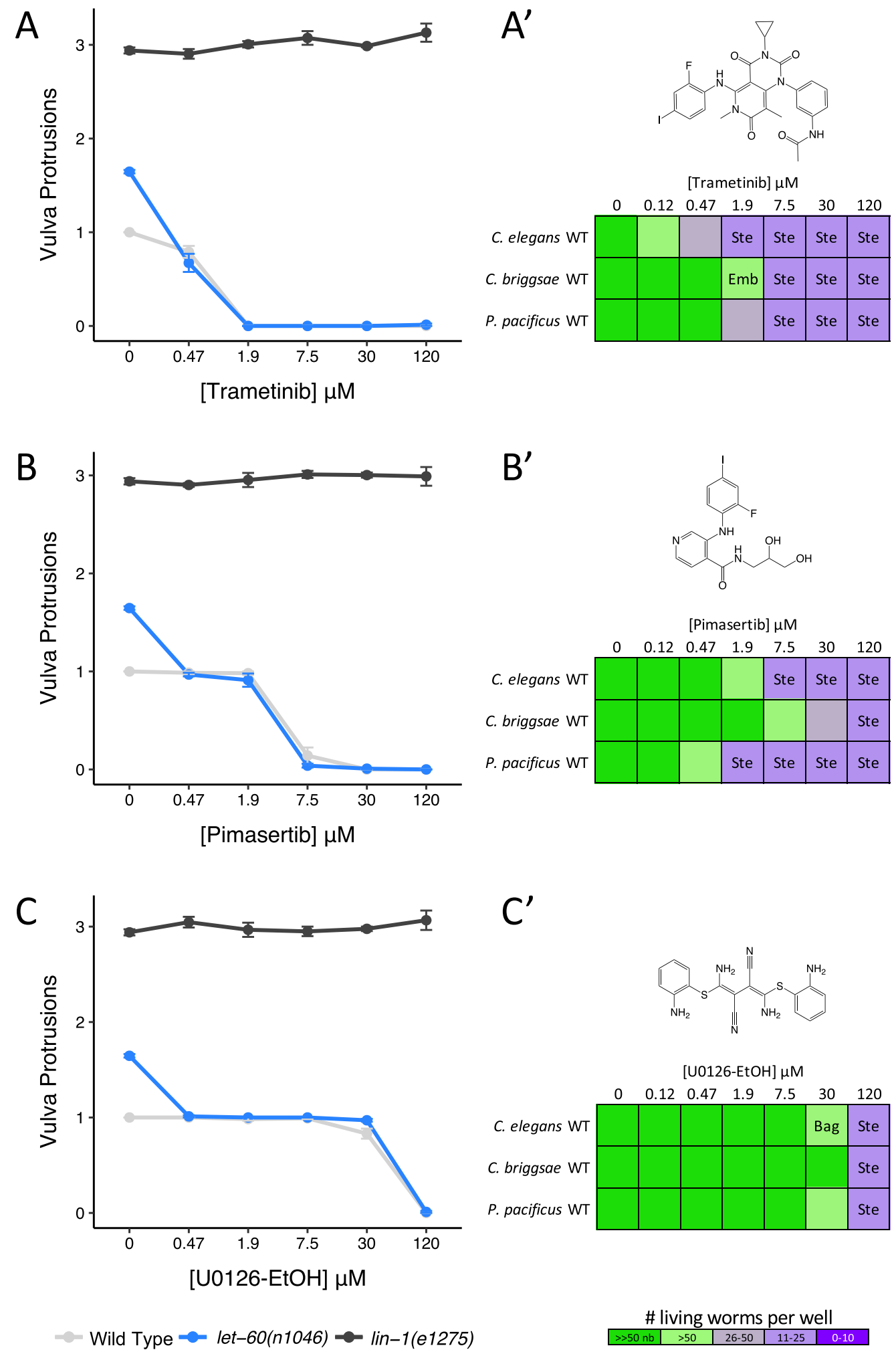

Figure 7. Vertebrate allosteric MEK inhibitors target worm MEK-2, inducing loss-of-function phenotypes across nematode species. (A-C) Three structurally distinct allosteric MEK inhibitors suppress the Multivulva phenotype of upstream let60(n1046) gain-of-function mutants, but have no effect on the Multivulva phenotype in downstream lin-1(e1275) loss-offunction mutants. The average number of vulva protrusions observed per worm in each condition quantified over 3 biological replicates is shown. Error bars indicate SEM. $\left(\mathbf{A}^{\prime}-\mathbf{C}^{\prime}\right)$ Phenotypes induced by allosteric MEK inhibitors in free-living nematode species C. elegans, C. briggsae and P. pacificus. Sterility (Ste), embryonic lethality (Emb) and bag-of-worms (Bag) phenotypes are reported along with the resulting population growth defects indicated by the colour coded scale (nb, no bacteria remaining in the well). 

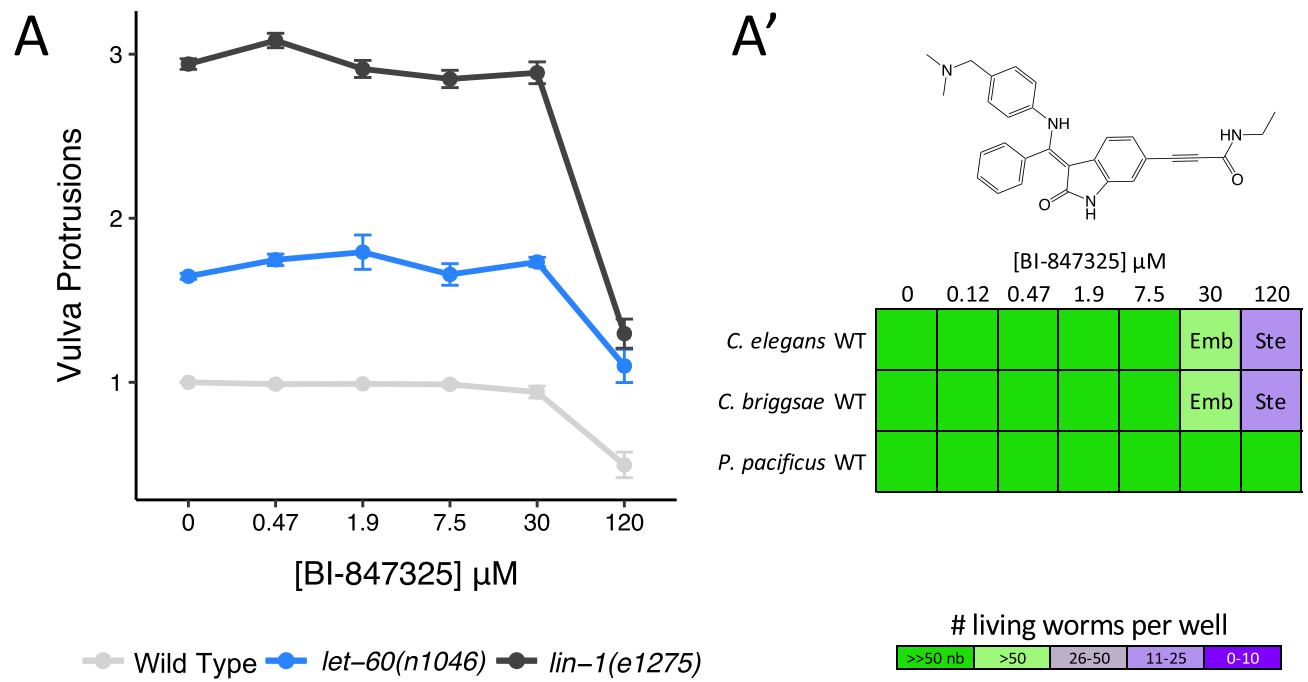

Figure 8. ATP-competitive MEK inhibitor BI-847325 does not engage nematode MEK-2. (A) The ATPcompetitive MEK inhibitor BI-847325 significantly suppresses vulva induction in Wild Type and both mutants at the highest concentration tested $(120 \mu \mathrm{M})$ relative to solvent controls (Student's T-test: $p<0.001)$. The average number of vulva protrusions observed per worm in each condition quantified over 3 biological replicates is shown. Error bars indicate SEM. (A') BI-847325 induces sterility (Ste) and embryonic lethality (Emb) phenotypes in C. elegans and C. briggsae resulting in the population growth defects indicated by the colour coded scale (nb, no bacteria remaining in the well).

compounds are likely hitting the expected target, we asked whether a strain carrying a weak loss-of-function allele of $p l k-1$ is hypersensitive to the compounds. We found that a strain carrying a temperature-sensitive allele of plk-1 (or683) exhibits twofold hypersensitivity to the 2,4-Dianilinopyrimidine GSK1520489 compared to the wild type control strain at the intermediate temperature of $20^{\circ} \mathrm{C}$ (Fig. 9A). The plk-1(or683) mutant showed an even greater sensitivity to the BT inhibitors, up to 32-fold relative to the wild type control. We assayed three of these BT inhibitors (GSK483724A, GSK479719A and GSK580432A) for their ability to inhibit C. elegans PLK-1 kinase activity in vitro and found the IC50 values for each of these inhibitors were in the low nanomolar range (91, 39 and $24 \mathrm{nM}$ respectively, Fig. 9B). Of those compounds that were available for retesting, the structurally related BT analogs GSK580432 and GSK479719A exhibit both the most potent activity in vivo across the nematode species tested and the most potent inhibition of Cel-PLK-1 kinase activity in vitro. These compounds may serve as valuable structures on which to base the generation of nematode-selective PLK-1 inhibitors.

Considering essential nematode-specific kinases as anthelmintic targets. An obvious alternative approach to the one we have taken here is to pursue essential nematode-specific kinases as anthelmintic targets. As described by Manning ${ }^{33}$, there are 15 nematode-specific kinase families within the C. elegans kinome, consisting of 105 nematode-specific kinase genes. Of these, disruptions of 16 lead to lethal, sterile, embryonic lethal or larval arrest phenotypes (WormBase, Supplementary Table 2). These nematode-specific essential kinases belong to families within the largely expanded CK1 group, the FGFR-like RTK family KIN-16 and three worm-specific families from the 'Other' group, which bear little resemblance to any vertebrate kinases ${ }^{33}$. Outside of the nematode-specific families, we identified 10 additional nematode-specific kinases with essential functions from families identified by Manning as expanded in nematodes ${ }^{33}$. These kinases primarily emerged from the expansion of the Fer kinase family in the worm ${ }^{33}$.

Of these 26 essential nematode-specific kinases, we find that 23 are more than $30 \%$ identical and/or align with over $70 \%$ of the kinase domain of the closest human kinase (Supplementary Table 2). The remaining three kinases K09E4.1, F54F7.5 (MES-1), and K09C6.8 do not have detectable orthologs encoded within many of the parasitic nematodes surveyed (Supplementary Fig. 4 K-M). We conclude that targeting nematode-specific kinases with the goal of developing a reasonably broad-spectrum anthelmintic is not a simple solution.

\section{Discussion}

Here, we have taken a small molecule screening approach to survey the C. elegans kinome and have identified 17 druggable essential nematode kinases (Table 1). In doing so, we have identified a suite of compounds that likely inhibit these kinases in vivo (Supplementary Table 1). Of these 17 druggable kinases, three (EGFR/LET-23, MEK1/MEK-2 and PLK1/PLK-1) are candidate anthelmintic targets because they harbor distinct amino acid residues in the presumptive drug-binding pocket when compared to the vertebrate ortholog (Table 1). These differences may be exploited to design small molecules that specifically and safely inhibit the essential nematode kinase within the context of an infected vertebrate host. 
A

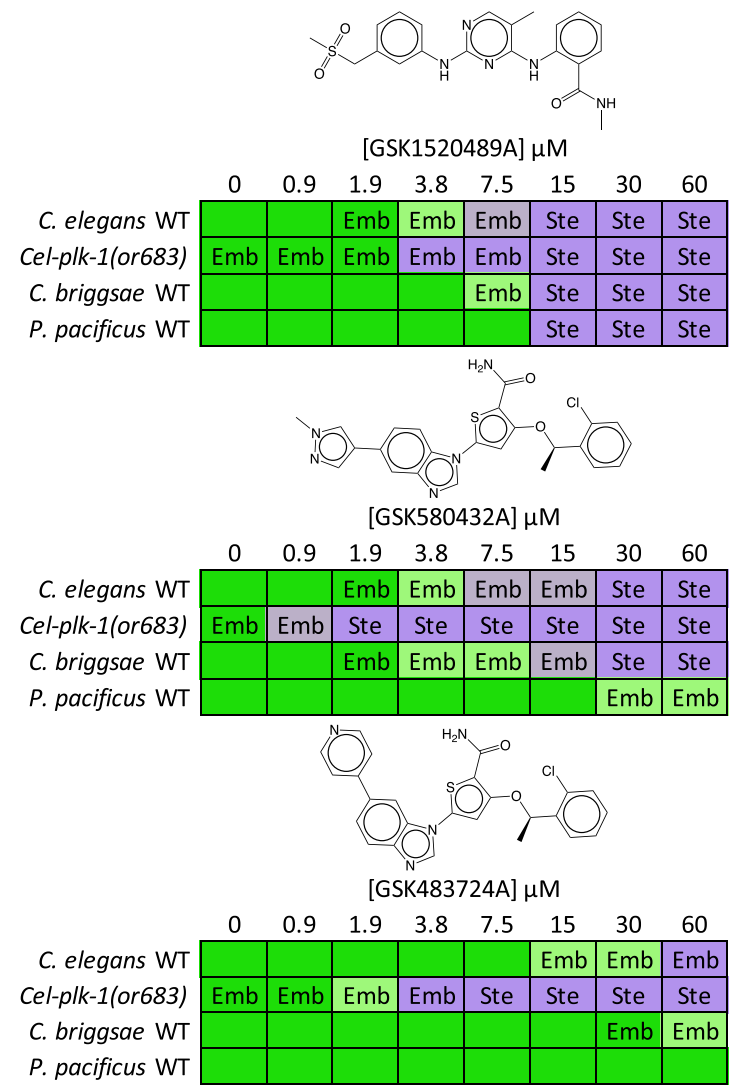

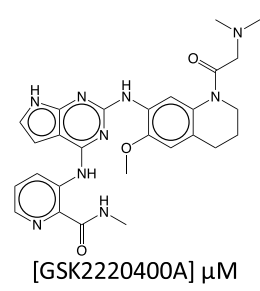

C. elegans WT

Cel-plk-1(or683)

C. briggsae WT

P. pacificus WT

\begin{tabular}{|c|c|c|c|c|c|c|c|}
\hline 0 & 0.9 & 1.9 & 3.8 & 7.5 & 15 & 30 & 60 \\
\hline & & Emb & Emb & Emb & Emb & Ste & Ste \\
\hline Emb & Emb & Emb & Emb & Emb & Emb & Ste & Ste \\
\hline & & & & & & & \\
\hline & & & & & & & \\
\hline
\end{tabular}

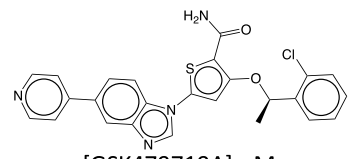

[GSK479719A] $\mu \mathrm{M}$

$\begin{array}{llllllll}0 & 0.9 & 1.9 & 3.8 & 7.5 & 15 & 30 & 60\end{array}$

C. elegans WT Cel-plk-1(or683)

C. briggsae WT P. pacificus WT
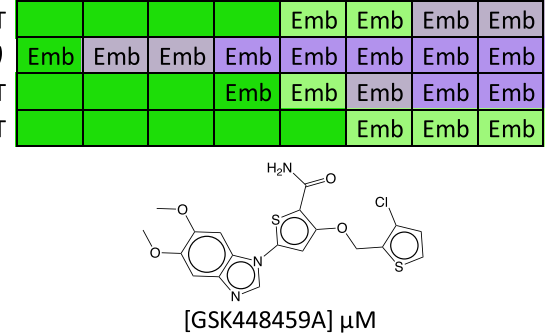

C. elegans WT Cel-plk-1(or683)

C. briggsae WT P. pacificus WT

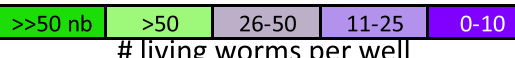

\# living worms per well

B

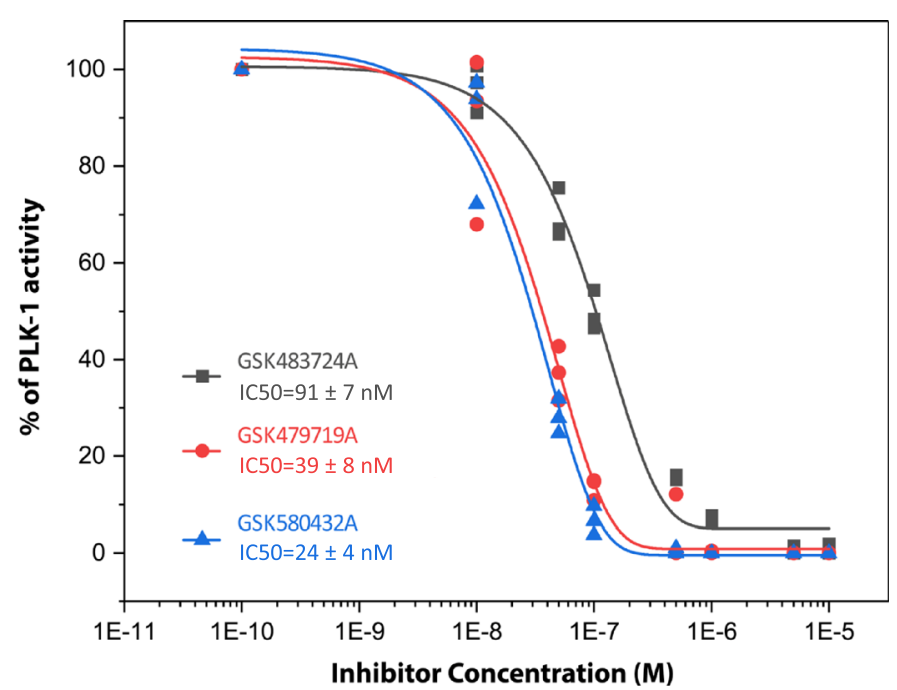

Figure 9. PLK1 inhibitors induce embryonic lethality and sterility phenotypes in nematodes. (A) PLK1 inhibitors from three core scaffolds including the 2,4-Dianilinopyrimidines (GSK1520489A), the 2,4-Dianilino pyrrolopyrimidines (GSK2220400A) and Benzimidazole N-thiophenes (GSK580432A, GSK479719A,

GSK483724A, and GSK448459A) induce phenotypes consistent with loss of C. elegans PLK-1 including sterility (Ste) and embryonic lethality (Emb) resulting in the population growth defects indicated by the colour coded scale (nb, no bacteria remaining in the well). (B) The three Benzimidazole N-thiophene PLK1 inhibitors tested (GSK483724A, GSK479719A and GSK580432A) inhibit C. elegans PLK-1 kinase activity in vitro with IC50 values of $91 \mathrm{nM}, 39 \mathrm{nM}$ and $24 \mathrm{nM}$ respectively. 
Our strategy to identify candidate anthelmintic targets began with identifying vertebrate kinase inhibitors that are active in whole-animal $C$. elegans assays. This allowed us to uncover both the nematode kinases that can be pharmacologically manipulated in vivo to produce deleterious effects as well as to identify the associated small molecule scaffolds that can reach their target in whole worms. These chemical scaffolds can be refined to increase specificity for the nematode ortholog of their target kinase.

Screens against any one nematode species, including C. elegans, are unlikely to reveal all compounds within a library that have anthelmintic potential. In other words, small molecule screens with C. elegans are bound to miss hits that would be found in screens with other nematode species. Furthermore, not all hits that disrupt $C$. elegans will translate to parasitic species ${ }^{65,66}$. However, there are significant advantages to using the free-living $C$. elegans nematode as a platform to identify candidate anthelmintic scaffolds. First, it is cost-effective and can be adapted to medium or high-throughput screens. Second, molecules found to be active against C. elegans have an increased likelihood of having activity against parasitic nematodes ${ }^{65,67}$. Third, C. elegans small molecule bioassays have proven to be exceptionally useful in identifying and/or characterizing several candidate anthelmintics ${ }^{65,68-72}$. Fourth, standard 6-day C. elegans bioassays of the type we have employed here probe the entire life cycle of the nematode. Hence, this approach can reveal nematode vulnerabilities that may not be accessible using bioassays with parasitic species, which are often limited to a single life stage. Finally, the wealth of genetic knowledge and C. elegans tools available allow us to make inferences about target engagement of bioactive molecules based on established genetic loss-of-function phenotypes, and can further help validate the target of these molecules with relative ease (this work) $)^{65,69,71,72}$.

Here, we have used C. elegans to demonstrate the anthelmintic potential of EGFR/LET-23, MEK1/MEK-2 and PLK1/PLK-1 kinase targets. There is corroborating evidence in the literature that supports the idea that these kinases may have value as anthelmintic targets. The EGFR/ERK pathway has been suggested as a therapeutic target in the tapeworm Echinococcus multilocularis, the causative parasite of alveolar echinococcosis ${ }^{15,73}$. In $E$. multilocularis, the EGFR/ERK signaling pathway has been implicated in promoting proliferation of the germinative cells, a stem cell-like population that drives larval growth and development. Exposure of E. multilocularis to the EGFR inhibitors CI-1033 and BIBW2992, or the MEK/ERK inhibitor U0126, impairs germinative cell proliferation, resulting in larval growth and development defects ${ }^{15}$. Furthermore, the E. multilocularis PLK1 homolog EmPlk1 is also expressed in the germinative cells and can be inactivated by the PLK1 inhibitor BI2536, inducing mitotic arrest and germinative cell death ${ }^{74}$.

Other studies have implicated the EGFR/ERK signaling pathway in the flatworm parasite Schistasoma mansoni as a target to treat schistosomiasis due to its role in the development of oocytes and the female somatic gonad ${ }^{75}$. A screen of the 114 compounds in the National Cancer Institute's Oncology Drug Set identified 11 compounds that had an effect on both $S$. mansoni adults and larvae in vitro with an $\mathrm{IC}_{50} \leq 10 \mu \mathrm{M}^{76}$. These included the MEK1/2 inhibitor trametinib and three tyrosine kinase inhibitors annotated to target EGFR (gefitinib, afatinib and vandetanib). Trametinib and vandetanib maintained activity in vivo and were found to reduce worm burden by $63.8 \%$ and $48.1 \%$ respectively in the chronic S. mansoni mouse model of infection after a single oral dose of $400 \mathrm{mg} / \mathrm{kg}^{76}$. Hence, nematode-selective EGFR and MEK inhibitors may have broad-spectrum utility.

PLK1 has also been investigated as an anthelmintic target in $S$. mansoni ${ }^{20}$. RNAi-knockdown of the S. mansoni PLK1 ortholog smPLK1 had a deleterious effect on post-infective larvae (schistosomulae). Small molecule inhibitors of human PLK1 were found to induce uncoordinated movements in adults and morphological defects in both schistosomulae and adult parasites. The benzimidazole thiophene core scaffold of PLK1 inhibitors from the GSK PKIS shows bioactivity in S. mansoni and was identified as a potential anti-schistosomal scaffold ${ }^{20}$. In the work presented here, we have also identified the benzimidazole thiophenes as a nematicidal scaffold that inhibits worm PLK-1, further reinforcing the idea that PLK-1 may be a useful anthelmintic target (Fig. 9; Supplementary File S2D).

The phenotypes induced by chemical inhibition of our three kinase targets of interest (LET-23, MEK-2 and PLK-1) include embryonic lethality, larval lethality or arrest, and sterility in C. elegans. Inhibiting these targets is unlikely to have the adulticidal effects needed for acute chemotherapeutic treatment for gastrointestinal (GI) nematode infections. However, the larvicidal effects of these inhibitors could in principle provide prophylactic protection against some species of GI parasites such as Ascaris lumbricoides or Ancylostoma duodenale by targeting larvae as they migrate throughout the body. In addition, pharmacological inhibition of these kinases could in principle be effective at combating human filarial nematode infections such as Onchocerca volvulus, which causes river blindness. River blindness is currently treated with ivermectin, which like the disruption of our kinase targets, kills larvae and reduces adult fertility ${ }^{77}$. Kinase inhibitors could be employed in parallel to ivermectin, which has a notoriously short half-life ${ }^{78}$.

A number of the kinase inhibitors that we found to have robust activity in C. elegans target vertebrate proteins that have no obvious ortholog in C. elegans (Supplementary Table 1). These hits include the 9 unique inhibitors that target vertebrate RET kinase and 17 unique inhibitors that target the vertebrate PDGFR family members PDGFRA, PDGFRB, KIT, FLT3 and CSF1R. The targets of other active inhibitors have a C. elegans ortholog, but these orthologs lack essential functions in C. elegans (Supplementary Table 1). For example, there are 16 unique compounds that inhibit mammalian VEGFR receptor family members FLT1, KDR and FLT4 that we found to elicit dramatic phenotypes in C. elegans. However, the corresponding C. elegans orthologs, VER-1, VER-3 and VER-4, have no reported phenotypes on WormBase, despite the characterization of both their genetic loss-offunction and RNAi phenotypes. The phenotypes elicited by these 16 compounds may be the result of promiscuous inhibition of multiple kinases or due to off-target effects. Deciphering the targets of these lethal compounds in C. elegans would be a valuable next step to uncover additional anthelmintic targets.

One alternative approach is to focus on developing inhibitors against nematode-specific kinases with no structurally similar match in vertebrate host species, such as the three we highlight here (K09E4.1, F54F7.5/ MES-1, and K09C6.8, Supplementary Table 2). There are two clear challenges associated with this alternative. 
First, identifying inhibitors that selectively target the nematode-specific kinases and do not inhibit a wide array of kinases (including those in the host) is a formidable challenge. In the approach that we have focused on here, extensive work has already gone into optimizing structures to selectively target EGFR, MEK1/MEK2, and PLK1. Second, and perhaps more importantly, nematode-specific kinases may be more evolutionarily divergent within Nematoda than those conserved with vertebrates (Supplementary Fig. 4) and it is unknown if the essential $C$. elegans function of any of these kinases is conserved with parasitic nematode species. Hence, any inhibitor that targets a given nematode-specific kinase may have limited utility against other nematode parasites.

Ample evidence shows that anthelmintic resistance is rampant among parasitic nematodes infecting livestock ${ }^{12}$, and resistance among human parasites is growing ${ }^{2}$. There is a clear need for the development of new anthelmintics to add to our arsenal. Here, we have highlighted three essential kinases that have good anthelmintic potential because of small sequence changes in the drug-binding pockets of the nematode orthologs relative to mammalian hosts. These kinases have proven to be druggable in whole worms, making them important targets for the development of novel anthelmintics.

\section{Methods}

Nematode strains and culture methods. Caenorhabditis elegans N2 (wild-type), CB1275 lin-1(e1275), C. briggsae AF16 (wild-type) and Pristionchus pacificus PS312 (wild-type) were provided by the Caenorhabditis Genetics Center (University of Minnesota). The JA337 strain let-23(sy1); jgIs19[1et-23p::LET-23::hEGFR-TK, rol-6(su1006)] was provided by Dr. Jaegal Shim. All animals were cultured using standard methods at $20^{\circ} \mathrm{C}^{39}$, with the exception of the temperature sensitive mutant plk-1(or683) which was cultured at $15^{\circ} \mathrm{C}$.

Chemical sources. The Library of Pharmacologically Active Compounds (Sigma-Aldrich) was purchased from the SickKids Proteomics, Analytics, Robotics \& Chemical Biology Centre (SPARC BioCentre). The APExBIO DiscoveryProbe Kinase Inhibitor Library was a gift from Jim Dowling. The OICR Kinase Inhibitor Library was a gift from Rima Al-Awar and David Uehling at the Ontario Institute of Cancer Research. The GSK Published Kinase Inhibitor Set (PKIS) molecules was obtained from GlaxoSmithKline. MEK Inhibitors trametinib, pimasertib, U0126-EtOH and BI-847325 and EGFR inhibitor gefitinib were purchased for further testing from Selleck Chemicals.

Kinase Inhibitor Screens. The kinase inhibitor screening method was adapted from previously described liquid-based screening protocols $s^{65}$ and is summarized in Fig. 1A. Briefly, Escherichia coli strain HB101 was resuspended in nematode growth media (NGM) buffered using Potassium Phosphate buffer $(\mathrm{pH}=6)^{79}$ or Citrate Phosphate buffer $(\mathrm{pH}=3)^{80}$. The final media $\mathrm{pH}$ for these two conditions were $\mathrm{pH}=7$ ("neutral" media) and $\mathrm{pH}=4.5$ ("acidic" media) respectively. The acidic media condition was included to improve our ability to capture phenotypes induced by molecules that are charged at neutral $\mathrm{pH}$ and thus may not be bioavailable to the worms. $40 \mu \mathrm{L}$ of bacterial suspension was dispensed into each well of a 96-well plate, and $300 \mathrm{~nL}$ of the small molecule inhibitors or of dimethyl sulfoxide (DMSO) vehicle control was pinned into the wells using a 96-well pinning tool (V\&P Scientific). Approximately 20 synchronized first-stage larvae (L1s) N2 worms obtained from an embryo preparation were then added to each well in $10 \mu \mathrm{L}$ M9 buffer (neutral media) or NGM (acidic media) $)^{79}$. The final concentration of DMSO in the wells was $0.6 \% \mathrm{v} / \mathrm{v}$. Plates were sealed with Parafilm and incubated for 6 days at $25^{\circ} \mathrm{C}$ while shaking at $200 \mathrm{rpm}$ (New Brunswick I26/I26R shaker, Eppendorf). On day 6 the plates were observed under a dissection microscope and embryonic lethality, sterility, larval arrest and lethality phenotypes were assessed in each condition.

The LOPAC library was screened once in its entirety in technical duplicate in both media conditions. Kinase inhibitor hits in either media condition identified in the primary screen were retested in duplicate in both media conditions. The OICR library was screened twice in its entirety in technical duplicate in both media conditions. The APExBIO library was screened in technical duplicate in neutral $\mathrm{pH}$ media only due to limited drug availability. The PKIS library was screened once in its entirety in technical duplicate in both media conditions. Hits in either media condition identified in the primary screen were retested in duplicate in both media conditions based on availability.

Hit compounds were defined by the following criteria: in the primary screens, compounds were considered hits if the inhibitor induced population growth defects by day 6 (both technical replicates have fewer than 50 worms, at least one of the two replicates has fewer than 25 worms) and/or relevant phenotypes of interest (Emb, Ste, Lva, Let, Bag, Rod-like progeny) in both technical replicates in either media condition. Inhibitors were included in the Supplementary File S1A 'Hit Summary' and considered hits for further analysis if: 1) upon retest, the inhibitor induced population growth defects (both technical replicates have fewer than 50 worms, at least one of the two replicates has fewer than 25 worms) and/or relevant phenotypes of interest (Emb, Bag, Ste, Lva, Let, Rod-like progeny) in both technical replicates as in the primary screen; 2) the inhibitor was considered a hit in the primary screen and we were not able to repeat due to insufficient molecule.

Follow up inhibitor dose-response analyses were performed using the same methodology described above using neutral media unless otherwise specified.

Kinase inhibitor target assignment. For the 'commercial' kinase inhibitor libraries (LOPAC, OICR and APExBIO) the library documentation included annotations for the kinases targeted by each inhibitor within the libraries. For the PKIS library, we used the published in vitro kinase inhibition data to assign kinase targets to each inhibitor hit ${ }^{35-37}$. For hits from PKIS1, any kinase that was inhibited $\geq 65 \%$ at $0.1 \mu \mathrm{M}$ compound concentration was considered a target. For hits from PKIS2, any kinase that was inhibited $\geq 90 \%$ at $1 \mu \mathrm{M}$ compound concentration was considered a target. If there were more than 10 kinase targets for a particular PKIS inhibitor 
according to these criteria, the inhibitor was annotated as having 'MANY' targets and the specific targets of these compounds were not included in the downstream analyses.

Homology modeling and sequence analysis. Caenorhabditis elegans orthologs of human kinases targeted by our inhibitors were identified using OrthoList $2^{81}$. C. elegans kinase protein structure homology models were generated using the SWISS-MODEL pipeline using the indicated structural templates accessed from the Protein Data Bank (PDB) ${ }^{41}$. At least two unique human kinase templates were used for modeling each nematode kinase ortholog if available. The structures were visualized using The PyMOL Molecular Graphic System (Version 2.1.1 Schrödinger, LLC). All residues lining the inhibitor binding pocket(s) for each kinase of interest were analyzed to identify divergent AA residues with side chains oriented towards the inhibitor binding site. Particular attention was given to those residues within close proximity to the bound inhibitor molecule (within $5 \AA$ as measured using PyMOL). Multiple sequence alignments of the kinase domains across vertebrate and nematode species were generated using Clustal Omega ${ }^{82}$. Relevant protein sequences from the following nematode and vertebrate species were identified using NCBI BLAST for inclusion in the alignments: free-living nematodes C. elegans (Clade V), C. briggsae (Clade V), P. pacificus (Clade V); parasitic nematodes Haemonchus contortus (Clade V), Necator americanus (Clade V), Ancylostoma duodenale (Clade V), Dictyocaulus viviparus (Clade V), Ancylostoma ceylanicum (Clade V), Strongyloides ratti (Clade IV), Brugia malayi (Clade III), Onchocerca ochengi (Clade III), Loa loa (Clade III), Trichuris trichiura or T. suis (Clade I); vertebrates Danio rerio, Gallus gallus, Mus musculus, Rattus norvegicus, Canis lupus familiaris, Ovis aries, Bos taurus and Homo sapiens.

Vulva phenotype analysis. Inhibitor exposure was performed in liquid media as described in the LiquidBased Kinase Inhibitor Screening method above. Analysis was performed once all F0 worms reached adulthood (Day 4). For the MEK inhibitor experiments, worms were mounted on a $2 \%$ agarose pad on a glass slide, observed using a $20 \times$ objective on a Leica DMRA microscope and the number of vulval protrusions were counted. For the EGFR inhibitor experiment, the adult worms were examined under a dissection scope and the vulva phenotype was categorized as Multivulva, WT or Vulvaless. F0 worms that contained hatched progeny trapped inside (bagof-worms phenotype) were assumed to not have a functioning vulva and were counted as Vulvaless. A minimum of three biological replicates were completed for all vulva phenotype analyses.

LET-23 protein expression and purification. LET-23 KD (866-1191) with an N-terminal 10xHis-tag was expressed and purified from Sf9 insect cells as previously described ${ }^{43}$, with the following exceptions. Purification buffers contained $25 \mathrm{mM}$ Tris- $\mathrm{HCl}$ at $\mathrm{pH}$ 8.5. LET-23 that was eluted from a $1 \mathrm{ml}$ His-Trap HP column was briefly concentrated, then further purified on a HiLoad Superdex $20016 / 600$ pg column (GE Healthcare) in buffer containing (50 mM Tris- $\mathrm{HCl}, \mathrm{pH} 8.5,150 \mathrm{mM} \mathrm{NaCl}, 2 \mathrm{mM}$ TCEP and 5\% glycerol). Protein was quickly concentrated, and flash frozen for assays.

LET-23 kinase activity assay. Kinase activity was measured in vitro using a continuous enzyme-coupled reaction system performed at $30 \mathrm{C}$ as previously described ${ }^{83}$. Reaction buffer contained $20 \mathrm{mM}$ Tris (pH 7.5), $10 \mathrm{mM} \mathrm{MgCl}$, and $100 \mu \mathrm{M}$ ATP. Poly-4Glu:Tyr peptide (Sigma-Aldrich) was used as the phosphorylation substrate at a concentration of $1 \mathrm{mg} / \mathrm{ml}$. Small unilamellar vesicles (SUV) containing DOPC and DOGS-NTA-Ni lipids (Avanti Polar Lipids) in a buffer containing $20 \mathrm{mM}$ Tris ( $\mathrm{pH} 7.5$ ) and $10 \mathrm{mM} \mathrm{MgCl} 2$ were produced by extrusion through a membrane containing $100 \mathrm{~nm}$ pores (Whatman) using a mini-Extruder apparatus. The total lipid concentration of 10X SUV stocks was fixed at $2 \mathrm{mg} / \mathrm{ml}$ with Ni-NTA-DGS lipid at 5 mol percent.

Compounds were diluted from DMSO stock solutions into either heated kinase buffer $(25 \mathrm{mM}$ Tris- $\mathrm{HCl}$, $\mathrm{pH} 7.5,20 \mathrm{mM} \mathrm{MgCl}$ ) or sodium acetate solution ( $\mathrm{pH} 4.0$ ), before further dilution into kinase buffer. Diluted compounds were pre-incubated with kinase and SUV for $\sim 30 \mathrm{~min}$ on ice before starting the reaction. Due to limited solubility of the compounds in aqueous buffers, the compounds may have partially adsorbed to the lipid micelles and hence the final concentration of available compound may be overestimated.

PLK-1 kinase activity assay. In vitro kinase assay was performed at $30^{\circ} \mathrm{C}$ in $10 \mu \mathrm{l}$ containing $50 \mathrm{mM}$ HEPES pH 7.6, $10 \mathrm{mM} \mathrm{MgCl}$, $1 \mu \mathrm{g}$ GST-LMN-1 Head fragment (1-47aa) as a substrate ${ }^{84}$, and $1 \mu \mathrm{M}$ C.e. PLK$1{ }^{85}$ with DMSO or inhibitors at different concentration as indicated. After 30 -min incubation at $30^{\circ} \mathrm{C}$, reactions were initiated by adding a mix of $0.2 \mathrm{mM}$ ATP and $5 \mu \mathrm{Ci} \gamma$ - $\left[{ }^{32} \mathrm{P}\right] \mathrm{ATP}$ for $13 \mathrm{~min}$ at $30^{\circ} \mathrm{C}$. Reactions were stopped by adding $5 \mu \mathrm{l}$ of Laemmli buffer and immediately boiled for $5 \mathrm{~min}$ at $95^{\circ} \mathrm{C}$. All the samples were loaded on 4-20\% SDS-PAGE (TGX Bio-Rad) and run in $1 \times$ Tris/glycine/SDS buffer. Proteins were detected using Stain Free technology and ChemiDoc MP Imaging System (Bio-Rad). Radioactivity was measured by Amersham Typhoon Imager (GE) and analyzed using ImageJ. Dose-response curve and IC50 calculations were constructed from the quantification of three replicates.

Received: 23 September 2020; Accepted: 8 April 2021

Published online: 28 April 2021

\section{References}

1. Pullan, R. L. et al. Global numbers of infection and disease burden of soil transmitted helminth infections in 2010. Parasit. Vectors 7,37 (2014). 
2. Schulz, J. D. et al. Preventive chemotherapy in the fight against soil-transmitted helminthiasis: Achievements and limitations. Trends Parasitol 34(7), 590-602 (2018).

3. Hotez, P. J. et al. Rescuing the bottom billion through control of neglected tropical diseases. Lancet 373(9674), 1570-1575 (2009).

4. DALYs, G.B.D. \& H. Collaborators. Global, regional, and national disability-adjusted life-years (DALYs) for 315 diseases and injuries and healthy life expectancy (HALE), 1990-2015: A systematic analysis for the Global Burden of Disease Study 2015. Lancet 388(10053), 1603-1658 (2016).

5. Coyne, D. L. et al. Plant-parasitic nematodes and food security in Sub-Saharan Africa. Annu. Rev. Phytopathol. 56, 381-403 (2018).

6. Kenyon, F. et al. Worm control in livestock: Bringing science to the field. Trends Parasitol. 33(9), 669-677 (2017).

7. Besier, B. New anthelmintics for livestock: The time is right. Trends Parasitol. 23(1), 21-24 (2007).

8. Hassan, M. A., Pham, T. H., Shi, H. \& Zheng, J. Nematodes threats to global food security. Acta. Agric. Scand. B. Soil Plant Sci. 63, $420-425$ (2013).

9. Geerts, S. \& Gryseels, B. Drug resistance in human helminths: Current situation and lessons from livestock. Clin. Microbiol. Rev. 13(2), 207-222 (2000).

10. Kaplan, R. M. Drug resistance in nematodes of veterinary importance: A status report. Trends Parasitol. 20(10), 477-481 (2004).

11. Sutherland, I. A. \& Leathwick, D. M. Anthelmintic resistance in nematode parasites of cattle: A global issue?. Trends Parasitol. 27(4), 176-181 (2011).

12. Kaplan, R. M. \& Vidyashankar, A. N. An inconvenient truth: Global worming and anthelmintic resistance. Vet. Parasitol. 186(1-2), 70-78 (2012).

13. Vercruysse, J. et al. Control of helminth ruminant infections by 2030. Parasitology 145(13), 1655-1664 (2018).

14. O'Connell, E. M. et al. Defining the target and the effect of imatinib on the filarial c-Abl homologue. PLoS Negl. Trop. Dis. 11(7), e0005690 (2017).

15. Cheng, Z. et al. EGF-mediated EGFR/ERK signaling pathway promotes germinative cell proliferation in Echinococcus multilocularis that contributes to larval growth and development. PLoS Negl. Trop. Dis. 11(2), e0005418 (2017).

16. Morel, M. et al. Compound library screening identified Akt/PKB kinase pathway inhibitors as potential key molecules for the development of new chemotherapeutics against schistosomiasis. Int. J. Parasitol. Drugs Drug Resist. 4(3), 256-266 (2014).

17. Gelmedin, V., Caballero-Gamiz, R. \& Brehm, K. Characterization and inhibition of a p38-like mitogen-activated protein kinase (MAPK) from Echinococcus multilocularis: Antiparasitic activities of p38 MAPK inhibitors. Biochem. Pharmacol. 76(9), 1068-1081 (2008).

18. Preston, S. et al. Low cost whole-organism screening of compounds for anthelmintic activity. Int. J. Parasitol. 45(5), 333-343 (2015).

19. Cheng, Z. et al. Impairing the maintenance of germinative cells in Echinococcus multilocularis by targeting Aurora kinase. PLoS Negl. Trop. Dis. 13(5), e0007425 (2019).

20. Long, T. et al. Structure-bioactivity relationship for benzimidazole thiophene inhibitors of Polo-Like Kinase 1 (PLK1), a potential drug target in Schistosoma mansoni. PLoS Negl. Trop. Dis. 10(1), e0004356 (2016).

21. Taylor, C. M. et al. Using existing drugs as leads for broad spectrum anthelmintics targeting protein kinases. PLoS Pathog. 9(2), e1003149 (2013).

22. Ardito, F. et al. The crucial role of protein phosphorylation in cell signaling and its use as targeted therapy (Review). Int. J. Mol. Med. 40(2), 271-280 (2017).

23. Gauthier, K. \& Rocheleau, C. E. C. elegans vulva induction: An in vivo model to study epidermal growth factor receptor signaling and trafficking. Methods Mol. Biol. 1652, 43-61 (2017).

24. Sundaram, M. V. Canonical RTK-Ras-ERK Signaling and Related Alternative Pathways 1-38 (WormBook, 2013).

25. Eswaran, J. \& Knapp, S. Insights into protein kinase regulation and inhibition by large scale structural comparison. Biochim. Biophys. Acta 1804(3), 429-432 (2010).

26. Berman, H. M. et al. The Protein Data Bank. Nucleic Acids Res. 28(1), 235-242 (2000).

27. Sanchez, R. \& Sali, A. Advances in comparative protein-structure modelling. Curr. Opin. Struct. Biol. 7(2), 206-214 (1997).

28. Xiang, Z. Advances in homology protein structure modeling. Curr. Protein Pept. Sci. 7(3), 217-227 (2006).

29. Fabbro, D. 25 years of small molecular weight kinase inhibitors: Potentials and limitations. Mol. Pharmacol. 87(5), 766-775 (2015).

30. Cohen, P. Protein kinases-The major drug targets of the twenty-first century?. Nat. Rev. Drug Discov. 1(4), 309-315 (2002).

31. Roskoski, R. Jr. Properties of FDA-approved small molecule protein kinase inhibitors: A 2020 update. Pharmacol. Res. 152, 104609 (2020).

32. Carles, F. et al. PKIDB: A curated, annotated and updated database of protein kinase inhibitors in clinical trials. Molecules 23(4), 908 (2018).

33. Manning, G. Genomic Overview of Protein Kinases 1-19 (WormBook, 2005).

34. Stroehlein, A. J. et al. The Haemonchus contortus kinome-a resource for fundamental molecular investigations and drug discovery. Parasit. Vectors 8, 623 (2015).

35. Drewry, D. H. et al. Progress towards a public chemogenomic set for protein kinases and a call for contributions. PLoS ONE 12(8), e0181585 (2017).

36. Drewry, D. H., Willson, T. M. \& Zuercher, W. J. Seeding collaborations to advance kinase science with the GSK Published Kinase Inhibitor Set (PKIS). Curr. Top. Med. Chem. 14(3), 340-342 (2014).

37. Elkins, J. M. et al. Comprehensive characterization of the Published Kinase Inhibitor Set. Nat. Biotechnol. 34(1), 95-103 (2016).

38. Tharmalingam, S. et al. Orthosteric and allosteric drug binding sites in the Caenorhabditis elegans mgl-2 metabotropic glutamate receptor. Neuropharmacology 63(4), 667-674 (2012).

39. Burns, A. R. et al. A predictive model for drug bioaccumulation and bioactivity in Caenorhabditis elegans. Nat. Chem. Biol. 6(7), 549-557 (2010).

40. Harris, T. W. et al. WormBase: A modern Model Organism Information Resource. Nucleic Acids Res. 48(D1), D762-D767 (2020).

41. Waterhouse, A. et al. SWISS-MODEL: Homology modelling of protein structures and complexes. Nucleic Acids Res. 46(W1), W296-W303 (2018).

42. Yun, C. H. et al. Structures of lung cancer-derived EGFR mutants and inhibitor complexes: Mechanism of activation and insights into differential inhibitor sensitivity. Cancer Cell 11(3), 217-227 (2007).

43. Liu, L. et al. Regulation of kinase activity in the Caenorhabditis elegans EGF receptor, LET-23. Structure 26(2), 270.e4-281.e4 (2018).

44. Chamberlin, H. M. \& Sternberg, P. W. The lin-3/let-23 pathway mediates inductive signalling during male spicule development in Caenorhabditis elegans. Development 120(10), 2713-2721 (1994).

45. Sternberg, P. W. et al. LET-23-mediated signal transduction during Caenorhabditis elegans development. Mol. Reprod. Dev. 42(4), 523-528 (1995).

46. Hill, R. J. \& Sternberg, P. W. The gene lin-3 encodes an inductive signal for vulval development in C. elegans. Nature 358(6386), 470-476 (1992).

47. Aroian, R. V. \& Sternberg, P. W. Multiple functions of let-23, a Caenorhabditis elegans receptor tyrosine kinase gene required for vulval induction. Genetics 128(2), 251-267 (1991).

48. Han, M. \& Sternberg, P. W. let-60, a gene that specifies cell fates during C. elegans vulval induction, encodes a ras protein. Cell 63(5), 921-931 (1990).

49. Yochem, J., Sundaram, M. \& Han, M. Ras is required for a limited number of cell fates and not for general proliferation in Caenorhabditis elegans. Mol. Cell Biol. 17(5), 2716-2722 (1997). 
50. Abdus-Saboor, I. et al. Notch and Ras promote sequential steps of excretory tube development in C. elegans. Development 138(16), 3545-3555 (2011).

51. Nelson, F. K., Albert, P. S. \& Riddle, D. L. Fine structure of the Caenorhabditis elegans secretory-excretory system. J. Ultrastruct. Res. 82(2), 156-171 (1983).

52. Nelson, F. K. \& Riddle, D. L. Functional study of the Caenorhabditis elegans secretory-excretory system using laser microsurgery. J. Exp. Zool. 231(1), 45-56 (1984).

53. Bae, Y. K. et al. An in vivo C. elegans model system for screening EGFR-inhibiting anti-cancer drugs. PLoS ONE 7(9), e42441 (2012).

54. Wu, Y., Han, M. \& Guan, K. L. MEK-2, a Caenorhabditis elegans MAP kinase kinase, functions in Ras-mediated vulval induction and other developmental events. Genes Dev. 9(6), 742-755 (1995).

55. Kornfeld, K., Guan, K. L. \& Horvitz, H. R. The Caenorhabditis elegans gene mek-2 is required for vulval induction and encodes a protein similar to the protein kinase MEK. Genes Dev. 9(6), 756-768 (1995).

56. Beitel, G. J., Clark, S. G. \& Horvitz, H. R. Caenorhabditis elegans ras gene let-60 acts as a switch in the pathway of vulval induction. Nature 348(6301), 503-509 (1990).

57. Beitel, G. J. et al. The Caenorhabditis elegans gene lin-1 encodes an ETS-domain protein and defines a branch of the vulval induction pathway. Genes Dev. 9(24), 3149-3162 (1995).

58. Dong, Q. et al. Discovery of TAK-733, a potent and selective MEK allosteric site inhibitor for the treatment of cancer. Bioorg. Med. Chem. Lett. 21(5), 1315-1319 (2011).

59. Sini, P. et al. Pharmacological profile of BI 847325 , an orally bioavailable, ATP-competitive inhibitor of MEK and aurora kinases. Mol. Cancer Ther. 15(10), 2388-2398 (2016).

60. Wu, P. K. \& Park, J. I. MEK1/2 inhibitors: Molecular activity and resistance mechanisms. Semin. Oncol. 42(6), 849-862 (2015).

61. McDowall, J. S. \& Rose, A. M. Genetic analysis of sterile mutants in the dpy-5 unc-13 (I) genomic region of Caenorhabditis elegans. Mol. Gen. Genet. 255(1), 60-77 (1997).

62. Chase, D. et al. The polo-like kinase PLK-1 is required for nuclear envelope breakdown and the completion of meiosis in Caenorhabditis elegans. Genesis 26(1), 26-41 (2000).

63. Nishi, Y. et al. Polo kinases regulate C. elegans embryonic polarity via binding to DYRK2-primed MEX-5 and MEX-6. Development 135(4), 687-697 (2008).

64. Kothe, M. et al. Selectivity-determining residues in Plk1. Chem. Biol. Drug Des. 70(6), 540-546 (2007).

65. Burns, A. R. et al. Caenorhabditis elegans is a useful model for anthelmintic discovery. Nat. Commun. 6, 7485 (2015).

66. Hahnel, S. R. et al. Caenorhabditis elegans in anthelmintic research-Old model, new perspectives. Int. J. Parasitol. Drugs Drug Resist. 14, 237-248 (2020).

67. Mathew, M. D. et al. Using C. elegans forward and reverse genetics to identify new compounds with anthelmintic activity. PLoS Negl. Trop. Dis. 10(10), e0005058 (2016).

68. Holden-Dye, L. \& Walker, R. J. Anthelmintic Drugs and Nematicides: Studies in Caenorhabditis elegans 1-29 (WormBook, 2014).

69. Driscoll, M. et al. Genetic and molecular analysis of a Caenorhabditis elegans beta-tubulin that conveys benzimidazole sensitivity. J. Cell Biol. 109(6 Pt 1), 2993-3003 (1989).

70. Schaeffer, J. M. et al. [3H]paraherquamide binding to Caenorhabditis elegans. Studies on a potent new anthelmintic agent. Biochem. Pharmacol. 43(4), 679-684 (1992).

71. Dent, J. A. et al. The genetics of ivermectin resistance in Caenorhabditis elegans. Proc. Natl. Acad. Sci. USA 97(6), 2674-2679 (2000).

72. Kaminsky, R. et al. A new class of anthelmintics effective against drug-resistant nematodes. Nature 452(7184), 176-180 (2008).

73. Gelmedin, V., Spiliotis, M. \& Brehm, K. Molecular characterisation of MEK1/2- and MKK3/6-like mitogen-activated protein kinase kinases (MAPKK) from the fox tapeworm Echinococcus multilocularis. Int. J. Parasitol. 40(5), 555-567 (2010).

74. Schubert, A. et al. Targeting Echinococcus multilocularis stem cells by inhibition of the Polo-like kinase EmPlk1. PLoS Negl. Trop. Dis. 8(6), e2870 (2014).

75. Andrade, L. F. et al. Regulation of Schistosoma mansoni development and reproduction by the mitogen-activated protein kinase signaling pathway. PLoS Negl. Trop. Dis. 8(6), e2949 (2014).

76. Cowan, N. \& Keiser, J. Repurposing of anticancer drugs: In vitro and in vivo activities against Schistosoma mansoni. Parasit. Vectors 8, 417 (2015).

77. Walker, M. et al. Macrofilaricidal efficacy of repeated doses of ivermectin for the treatment of river blindness. Clin. Infect. Dis. 65(12), 2026-2034 (2017).

78. Gonzalez Canga, A. et al. The pharmacokinetics and interactions of ivermectin in humans-A mini-review. AAPS J. 10(1), 42-46 (2008).

79. Stiernagle, T., Maintenance of C. elegans. WormBook, 2006: p. 1-11.

80. Mohan, C. Buffers. A Guide for the Preparation and Use of Buffers in Biological System (EMD Bioscience, 2006).

81. Kim, W. et al. OrthoList 2: A new comparative genomic analysis of human and Caenorhabditis elegans genes. Genetics 210(2), 445-461 (2018).

82. Sievers, F. et al. Fast, scalable generation of high-quality protein multiple sequence alignments using Clustal Omega. Mol. Syst. Biol. 7, 539 (2011).

83. Zhang, X. et al. An allosteric mechanism for activation of the kinase domain of epidermal growth factor receptor. Cell 125(6), 1137-1149 (2006).

84. Velez-Aguilera, G. et al. PLK-1 promotes the merger of the parental genome into a single nucleus by triggering lamina disassembly. Elife https://doi.org/10.7554/eLife.59510 (2020).

85. Tavernier, N. et al. Cdk1 phosphorylates SPAT-1/Bora to trigger PLK-1 activation and drive mitotic entry in C. elegans embryos. J. Cell Biol. 208(6), 661-669 (2015).

86. Wood, E. R. et al. A unique structure for epidermal growth factor receptor bound to GW572016 (Lapatinib): Relationships among protein conformation, inhibitor off-rate, and receptor activity in tumor cells. Cancer Res. 64(18), 6652-6659 (2004).

\section{Acknowledgements}

The Ontario Institute for Cancer Research (OICR) Kinase Inhibitor Library was provided by Rima Al-Awar and David Uehling at OICR. The APExBIO DiscoveryProbe Kinase Inhibitor Library was provided by Jim Dowling. The GSK Published Kinase Inhibitor Set (PKIS) molecules were provided by William Zuercher. We are grateful for the gift of the humanized LET-23 C. elegans strain from Dr. Jaegal Shim and to Prof. Tim Geary for helpful conversations. Some C. elegans strains were provided by the CGC, which is funded by NIH Office of Research Infrastructure Programs (P40 OD010440). This work is supported by a CIHR grant (313296) and a Canada Research Chair in Chemical Genetics to PJR, an NSERC Alexander Graham Bell Canada Graduate Scholarship to JK, an NIH grant (R01GM109176) to N.Jura, an Association pour la Recherche sur le Cancer Project Fellowship and funding from "Dynamic Research" from Agence Nationale de la Recherche (ANR-18-IDEX-0001), Université de Paris excellency initiative (IdEx) to N. Joly, and work in the laboratory of L.P. is supported by the 
French Agence Nationale de la Recherche under grant no. ANR-17-CE13-0011-01/01, by Ligue Contre le Cancer (Programme Equipes Labellisées).

\section{Author contributions}

J.K. and P.J.R. conceived of the project, experiments, and wrote the manuscript. J.K. performed all experiments except the in vitro kinase assays, and prepared all figures, tables, and supplementary files. W.Z. provided the PKIS libraries and consulted on experiments and manuscript edits. N.Joly and L.P. conducted the PLK-1 kinase inhibition assays. E.M.L., J.A.C-N., and N.Jura conducted the LET-23 kinase inhibition assays. All authors reviewed the manuscript.

\section{Competing interests}

The authors declare no competing interests.

\section{Additional information}

Supplementary Information The online version contains supplementary material available at https://doi.org/ 10.1038/s41598-021-88150-6.

Correspondence and requests for materials should be addressed to P.J.R.

Reprints and permissions information is available at www.nature.com/reprints.

Publisher's note Springer Nature remains neutral with regard to jurisdictional claims in published maps and institutional affiliations.

(c) (i) Open Access This article is licensed under a Creative Commons Attribution 4.0 International License, which permits use, sharing, adaptation, distribution and reproduction in any medium or format, as long as you give appropriate credit to the original author(s) and the source, provide a link to the Creative Commons licence, and indicate if changes were made. The images or other third party material in this article are included in the article's Creative Commons licence, unless indicated otherwise in a credit line to the material. If material is not included in the article's Creative Commons licence and your intended use is not permitted by statutory regulation or exceeds the permitted use, you will need to obtain permission directly from the copyright holder. To view a copy of this licence, visit http://creativecommons.org/licenses/by/4.0/.

(C) The Author(s) 2021 